\title{
Exploring Structural Consistency in Graph Regularized Joint Spectral-Spatial Sparse Coding for Hyperspectral Image Classification
}

\author{
Changhong Liu, Jun Zhou, Senior Member, IEEE, Jie Liang, Yuntao Qian, Member, IEEE, Hanxi Li, \\ and Yongsheng Gao, Senior Member, IEEE
}

\begin{abstract}
In hyperspectral image classification, both spectral and spatial data distributions are important in describing and identifying different materials and objects in the image. Furthermore, consistent spatial structures across bands can be useful in capturing inherent structural information of objects. These imply that three properties should be considered when reconstructing an image using sparse coding methods. First, the distribution of different ground objects leads to different coding coefficients across the spatial locations. Second, local spatial structures change slightly across bands due to different reflectance properties of various object materials. Finally and more importantly, some sort of structural consistency shall be enforced across bands to reflect the fact that the same object appears at the same spatial location in all bands of an image. Based on these considerations, we propose a novel joint spectral-spatial sparse coding model that explores structural consistency for hyperspectral image classification. For each band image, we adopt a sparse coding step to reconstruct the structures in the band image. This allows different dictionaries be generated to characterize the band-wise image variation. At the same time, we enforce the same coding coefficients at the same spatial location in different bands so as to maintain consistent structures across bands. To further promote the discriminating power of the model, we incorporate a graph Laplacian sparsity constraint into the model to ensure spectral consistency in the dictionary generation step. Experimental results show that the proposed method outperforms some state-of-the-art spectral-spatial sparse coding methods.
\end{abstract}

Index Terms-Graph Laplacian regularizer, hyperspectral image, sparse coding, structural consistency.

Manuscript received April 15, 2016; revised July 9, 2016 and August 10, 2016; accepted August 11, 2016. Date of publication September 8, 2016; date of current version February 13, 2017. This work was supported by the Australian Research Council Linkage under Project LP150100658, the National Natural Science Foundation of China under Grant 61571393, Grant 61462042, Grant 61365002, and Grant 61262036, and the Visiting Scholars Special Funds from Young and Middle-aged Teachers Development Program for Universities in Jiangxi Province. (Corresponding author: Jun Zhou.)

C. Liu and H. Li are with the School of Computer and Information Engineering, Jiangxi Normal University, Nanchang 330022, China (e-mail: ahong286@ 126.com; hanxi.li@nicta.com.au).

J. Zhou is with the School of Information and Communication Technology, Griffith University, Brisbane, QLD 4111, Australia (e-mail: jun.zhou@ griffith.edu.au).

J. Liang is with the School of Engineering, Australian National University, Canberra, ACT 0200, Australia (e-mail: jie.liang@ anu.edu.au).

Y. Qian is with the Institute of Artificial Intelligence, College of Computer Science, Zhejiang University, Hangzhou 310027, China (e-mail: ytqian@zju.edu.cn; yuntaoq@yahoo.com.cn).

Y. Gao is with the School of Engineering, Griffith University, Brisbane, QLD 4111, Australia (e-mail: yongsheng.gao@ griffith.edu.au).

Color versions of one or more of the figures in this paper are available online at http://ieeexplore.ieee.org.

Digital Object Identifier 10.1109/JSTARS.2016.2602305

\section{INTRODUCTION}

$\mathbf{R}$ EMOTE sensing hyperspectral images (HSI) are acquired in hundreds of bands to measure the reflectance of earth surface, discriminate various materials, and classify ground objects. HSI classification aims at assigning each pixel with one thematic class in a scene [1]. Various machine learning models have been proposed for this purpose, such as Bayesian model [1], random forest [2], neural networks [3], support vector machines (SVM) [4]-[7], sparse representation [8]-[13], and deep learning [14], [15].

Many HSI classification methods make prediction based on the spectral response at a single pixel [6], [8], [9], [16]-[19]. While spectral information is essential in image classification and material identification, information extracted from spatial domain is very useful to discriminate various targets made of the same materials [20], [21]. To address this need, spectralspatial HSI classification approaches have been reported, each type of approach exploring and exploiting different ways to integrate spatial features with spectral features. Mura et al. and Ghamisi et al. proposed mathematical morphology methods to analyze spatial relationships between pixels using structured elements [22], [23]. Markov random field methods considered spatial information by adding to the objective function a term that defines spatial correlations in the prior model [24], [25]. Qian et al. developed three-dimensional (3-D) discrete wavelet transform to extract 3-D features along spectral and spatial dimensions simultaneously [26]. Moreover, many researchers proposed sparse representation methods to include spatial sparsity constraints or Kernel function to integrate spectral and spatial features [10], [12], [27]-[31].

Among these approaches, sparse representation-based classifiers have achieved the state-of-the-art performance [27], [32]. They provide an effective way of modeling the spatial neighborhood relationship and the distribution of atoms in the spectral or spatial domain, so that both spectral and spatial information can be seamlessly integrated and modeled. In sparse representation, a test sample is treated as a linear combination of atoms from training samples or a learned dictionary. A sparse regularization term is normally included to learn a discriminative representation of images [33]-[35]. Recently, structured sparsity priors are also incorporated into reconstruction methods [12], [36]-[39]. These include joint sparsity constraint [40], group sparsity constrain [41], graph 
Band 6
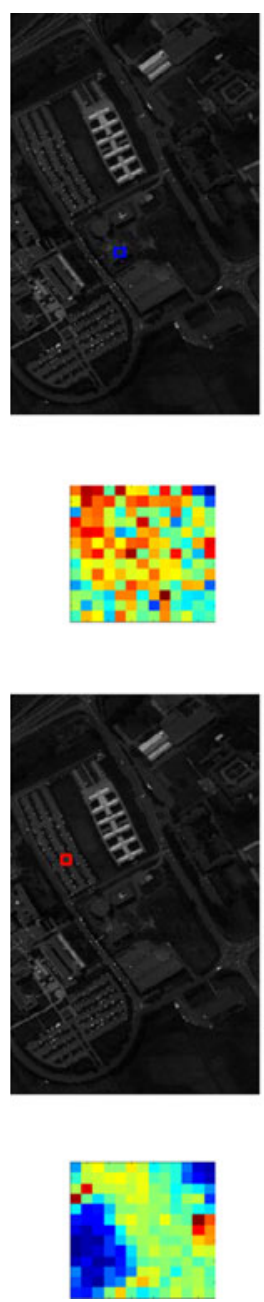

Band 15
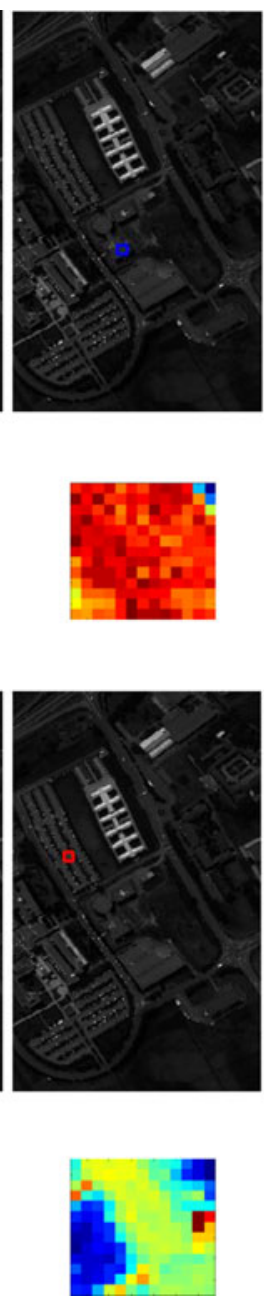

Band 32
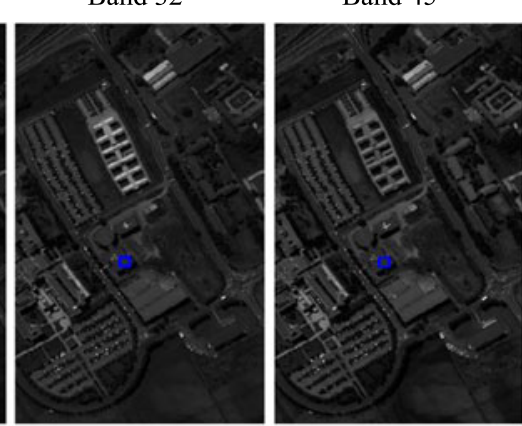

(a)
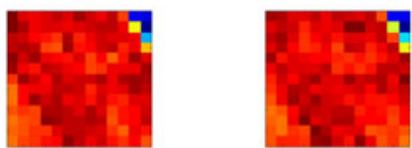

(b)
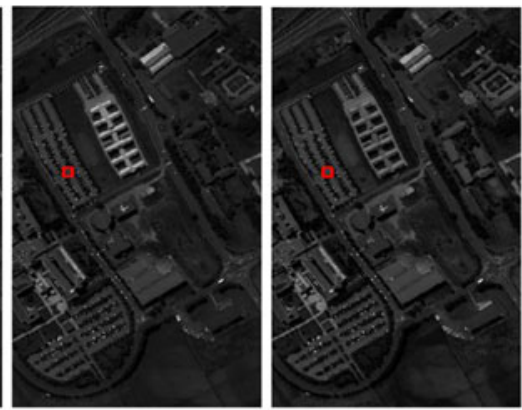

(c)
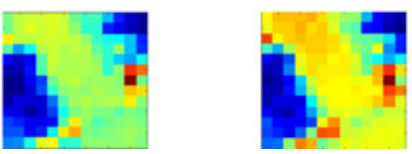

Band 80
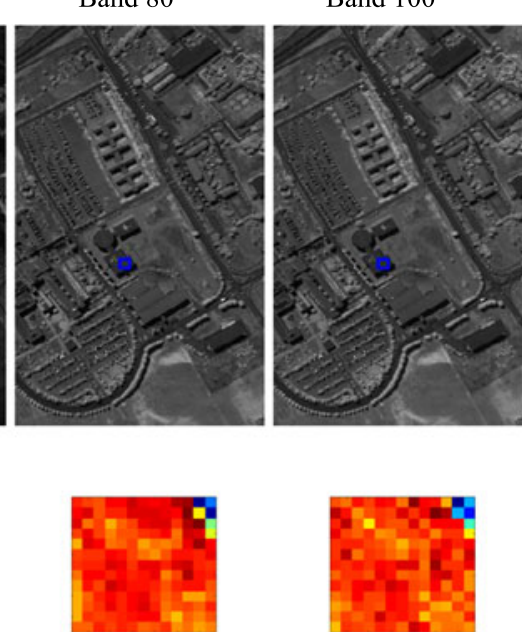
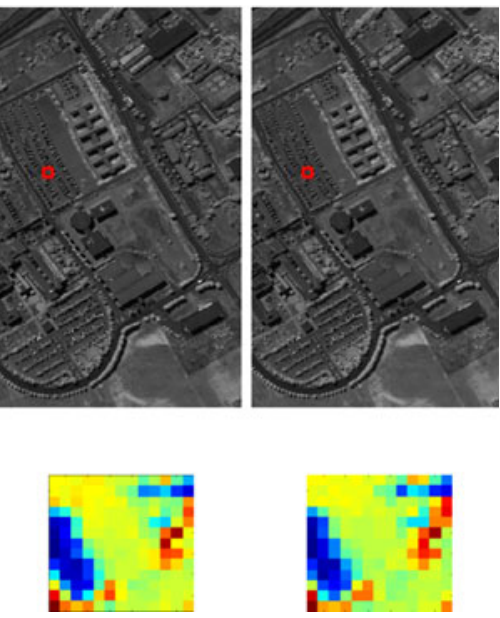

(d)

Fig. 1. Consistent structures in the University of Pavia dataset. (a) Pixel $(362,149)$ in bands $6,15,32,45,80$, and 100 for the "Bitumen" class. (b) $13 \times 13$ patches centered at pixel $(362,149)$. (c) Pixel $(251,76)$ in bands $6,15,32,45,80$, and 100 for the "Bricks" class. (d) $13 \times 13$ patches centered at pixel $(251,76)$.

Laplacian sparsity constraint [36], low-rank constraint [42], and low-rank group sparsity constraint [12]. Graph Laplacian sparsity constraint is based on the spatial dependencies between the neighboring pixels [12], [36]. It preserves the local manifold structure so that if two data points are close in their original data space, the sparse representations of these two data points are also close to each other in the new data space.

Although spectral-spatial analysis has been studied intensively in HSI classification, how to explore the local structural information has not been adequately addressed. To get a deeper understanding of the structural information embedded in a hyperspectral image, we use the University of Pavia image in Fig. 1 as an example. In the first row, bands $6,15,32,45,80$, and 100 are displayed. In the second and fourth rows, small patches extracted from neighborhoods around pixels $(362,149)$ and $(251,76)$ are displayed, respectively. Three observations can be obtained from this figure. First, various land cover classes have different distributions in the spatial domain. This happens in all bands. Second, local spatial structures change slightly across different bands. This is due to the distinct reflectance properties of object materials at different light wavelength. As a consequence, the extracted Gabor features at the same location in different bands also change slightly. Finally and more importantly, some sort of structural consistency can be observed across bands, as the ground objects at each location are consistent in all bands. Such consistency has been proved to be useful in hyperspectral image denoising [43]. In general, image representation and classification models shall be able to address all these observations.

Motivated by the above observations, we propose a novel joint spectral-spatial framework to explore structural consistency along all bands in the sparse coding model. For each band image, image patches or 2-D image features centered at pixels are first extracted from local neighborhood, which contain local structures of the central pixels. Then a sparse coding step is adopted to reconstruct the structures in the band images. This allows different dictionaries to be generated to characterize the band-wise image variation. At the same time, 
consistent structures across bands are maintained by enforcing the same coding coefficients at the same spatial location in different bands. To further promote the discriminating power of the model, a graph Laplacian sparsity constraint is incorporated into the model to ensure spectral consistency in the dictionary generation step. At last, the learned coefficients are fed into the classifier for pixel-wise classification.

The contribution of this paper lies in two aspects. First, we propose a novel joint spectral-spatial sparse coding framework, which can explore the structural consistency along all bands and integrate the spectral information and spatial structures into a sparse coding framework. Under this framework, 2-D structural features can be applied to HSI classification effectively and directly, and the learned coefficients inherently contain both spectral characteristics and spatial structures of HSI images. Second, we extend this model by including a graph regularization term to preserve the spectral relations between data points. This allows better relationship between data to be modeled, which improves the classification performance.

The rest of this paper is organized as follows. In Section II, we review related work on sparse representation-based hyperspectral image classification. In Section III, we first briefly introduce the basic sparse coding model. Then we describe the proposed method that preserves structural consistency, its graph-based extension, and the optimization algorithm for learning sparse coefficient and dictionaries. Experimental results are presented in Section IV. We conclude our work and point out future research direction in Section V.

\section{RELATED WORK}

Recently, sparse representation has been widely used in HSI classification. It allows spectral information be combined with spatial information, so that discriminative image representation can be achieved. Some of them directly extract the spectralspatial features and then feed these features into the sparse representation model. Qian et al. extracted a 3-D discrete wavelet transform (3-D-DWT) texture features to capture geometrical and statistical spectral-spatial structures and then applied them to the sparse representation model [26]. He et al. proposed an $l_{1}$ minimization-based spectral-spatial classification method. A spatial translation invariant wavelet sparse representation [13] was adopted in the model. Yang et al. combined Gabor spatial features and nonparametric weighted spectral features, and then applied sparse representation to describe the HSI [11]. To selected the most representative Gabor cube features for image classification, Jia et al. proposed to use Fisher discrimination criterion and a multitask joint sparse representation framework [31].

Structured sparsity constraints [12], [36], [40]-[42] are often incorporated into sparse representation to improve the performance of HSI classification. These methods explore the spatial dependencies between neighboring pixels, or the inherent structure of dictionary, or both [12]. For example, Laplacian constraint has been incorporated into the sparse recovery optimization problem such that the reconstructed neighboring pixels have similar spectral characteristics [44]. In [26], Qian et al. used sparsity constraints to help selecting the discriminant features from a pool of 3-D-DWT texture features. Low rank sparse representation methods have also been proposed to explore the spatial correlations of neighboring pixels [12], [45].

The sparse representation is further extended into kernelized or joint sparse form to exploit the spatial correlation across neighboring pixels. Chen et al. used simultaneous subspace pursuit method, simultaneous orthogonal matching pursuit (SOMP), and their kernelized extension for spectral-spatial HSI classification [10], [44]. He et al. utilized empirical mode decomposition and morphological wavelet transform to extract spectral-spatial features, which were then integrated by a sparse multitask learning method [28]. Liu et al. proposed a neighboring filtering Kernel sparse representation for enhanced classification of HSIs [27]. The relationship between pixels in a local neighborhood can also be modeled by structural similarity [29], graph embedding [32], and set-to-set distance [46]. When joint sparse representation is concerned, Zhang et al. constructed multiple features and enforced pixels in a small region to share the same sparsity pattern for each type of feature [47]. Wang et al. presented a spatial-spectral derivative-aided Kernel joint sparse representation, which considered high order spatial context and distinct spectral information [30].

Most aforementioned methods combine spectral information with spatial information using spatial constraints, complex spectral-spatial features, or joint sparse form of neighborhood pixels, and then make classification by minimizing the residual between a testing sample and the reconstructed image. Unlike the sparse representation procedure, Farani and Rabiee [48] proposed a sparse coding method for HSI classification, which used a spatially weighted sparse unmixing approach as a front-end and the learned sparse codes as the input to a linear SVM. Similar to this work, in our method, we also use learned sparse codes as inputs to the the classifier. This is done by first extracting 2-D spatial features from local neighborhoods and the learning band-wise distinct dictionary. Finally, we force the same spatial location in different bands share the same coding coefficients to capture the structural consistency along all bands.

\section{SCSC FOR HSI CLASSIFICATION}

In an HSI, the distributions of spatial structures vary in different spatial locations, but are closely related to each other in different bands. Such structural consistency has been shown in Fig. 1. Considering the spatial correlation along the spectral dimension, we construct a joint spectral-spatial sparse coding model with structural consistency by assigning the same coefficient in the same position of different bands. The modeling process also preserves the distinct band characteristics by producing band-wise dictionaries. The framework of the proposed SCSC method is shown in Fig. 2.

At each pixel, local spatial feature is first extracted band by band. Though any type of 2-D feature can be used here, we adopted a simple solution by extracting image patch centered at the pixel. Then, the dictionaries on all bands are trained individually and simultaneously with the same coefficient across bands. That is to say, the $b$ th dictionary is learned only using the spatial 


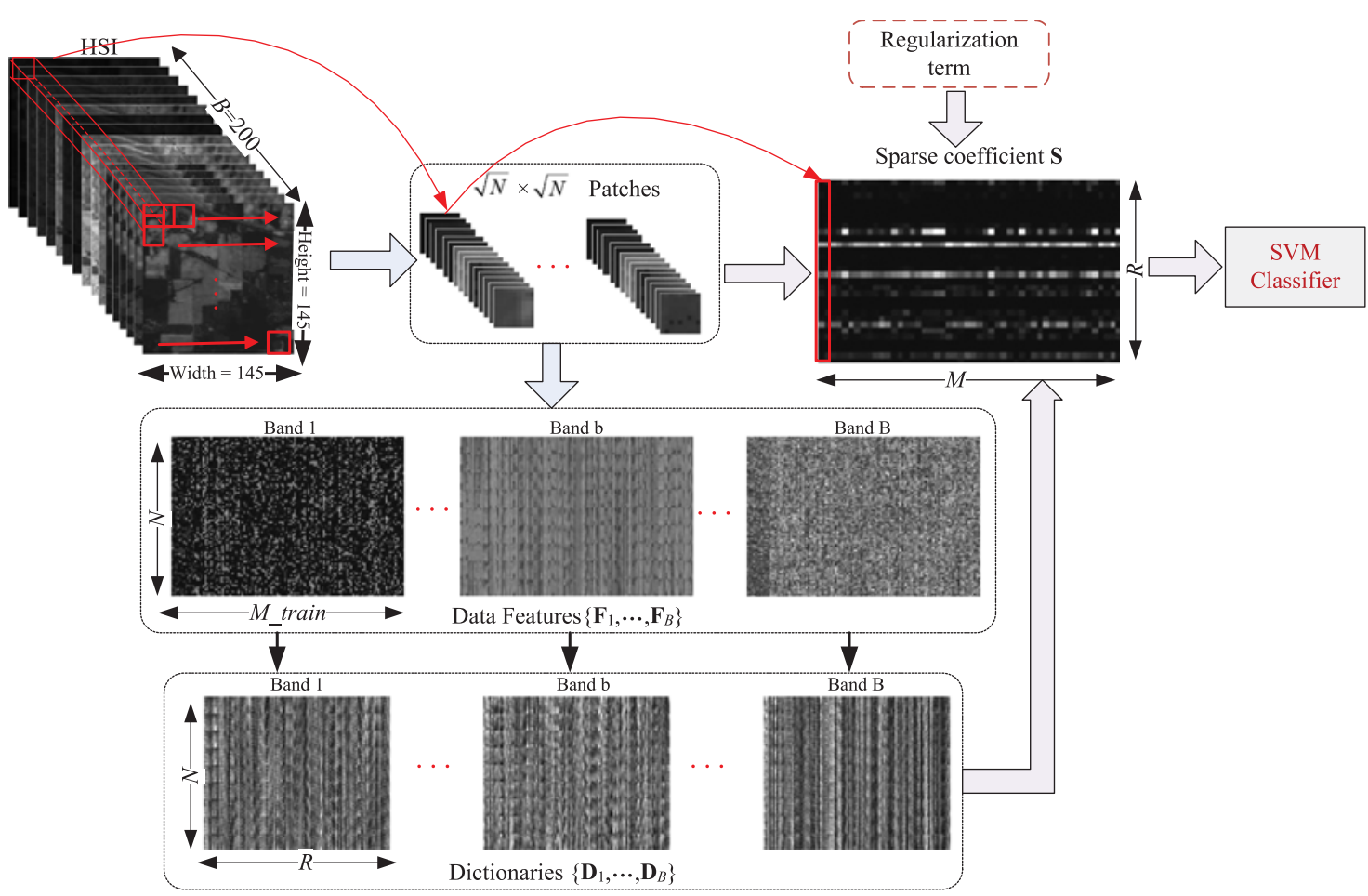

Fig. 2. Framework of the proposed method. $M$ is the total number of samples and $M_{-}$train is the number of training samples to train the dictionaries.

features extracted from the $b$ th band and there are $B$ different dictionaries for $B$ bands of HSI. These learned dictionaries are then used to estimate a sparse coefficient for each pixel. The dictionary and coefficient are optimized iteratively until convergence. Finally, the sparse coefficients are fed into SVM for classifier learning or classification. The whole process includes two stages: training and testing. In the training stage, the dictionaries are learned using the training samples without using the class label information. Then, the sparse coefficients of these samples are calculated and used to train the SVM classifier on labelled training samples. In the testing stage, the sparse coefficients are first calculated for the testing samples using the learned dictionaries and are then fed into the SVM classifier for prediction.

\section{A. Sparse Coding for Single-band Image}

An HSI is a 3-D structure and each band is a 2-D image, so the sparse coding model of single band image can be generated following the general image sparse coding methods. In order to describe the spatial relationships between neighbourhoods, a $\sqrt{N} \times \sqrt{N}$ patch is extracted at each pixel and then reshaped into an $N \times 1$ vector. Then, the $b$ th band HSI can be denoted as $\mathbf{F}_{b}=\left[\mathbf{f}_{b 1}, \ldots, \mathbf{f}_{b M}\right] \in \mathbb{R}^{N \times M}$ where $M$ denotes the number of pixels. Let $\mathbf{D}_{b}=\left[\mathbf{d}_{b 1}, \ldots, \mathbf{d}_{b R}\right] \in \mathbb{R}^{N \times R}$ be the dictionary matrix of the $b$ th band, where each $\mathbf{d}_{b i}$ denotes a basis vector in the dictionary, and $R$ is the number of basis vector. Let $\mathbf{S}_{b}=$ $\left[\mathbf{s}_{b 1}, \ldots, \mathbf{s}_{b M}\right] \in \mathbb{R}^{R \times M}$ be the coefficient matrix, where each column is a sparse coefficient for a pixel. The neighborhood feature of each pixel $\mathbf{f}_{b i}$ can be represented as a sparse linear combination of the basis vectors in the dictionary. The sparse coefficient of $\mathbf{f}_{b i}$ can be obtained by minimizing the loss function with an $\ell_{1}$ regularizer as follows:

$$
\begin{aligned}
& w \min _{\mathbf{D}_{b}, \mathbf{s}_{b i}}\left\|\mathbf{f}_{b i}-\mathbf{D}_{b} \mathbf{s}_{b i}\right\|^{2}+\beta\left\|\mathbf{s}_{b i}\right\|_{1} \\
& \text { s.t. }\left\|\mathbf{d}_{b i}\right\| \leq c, i=1, \ldots, R
\end{aligned}
$$

where $\beta$ is the regularization parameter controlling the degree of sparsity and $c$ is a constant. By summing up the loss functions at each pixel, we can formulate the objective function of the $b$ th band image as

$$
\begin{aligned}
& \min _{\mathbf{D}_{b}, \mathbf{S}_{b}}\left\|\mathbf{F}_{b}-\mathbf{D}_{b} \mathbf{S}_{b}\right\|^{2}+\beta\left\|\mathbf{S}_{b}\right\|_{1} \\
& \text { s.t. }\left\|\mathbf{d}_{b i}\right\| \leq c, i=1, \ldots, R .
\end{aligned}
$$

This problem can be solved by alternatively optimizing basis vectors $\mathbf{D}_{b}$ and coefficients $\mathbf{S}_{b}$ while fixing the other. For $\mathbf{S}_{b}$, the optimization can be solved by optimizing over each coefficient $\mathbf{s}_{b i}$ individually. This is an $\ell_{1}$ norm regularized linear regression problem, so it can be solved efficiently by many optimization algorithms such as the feature-sign algorithm [49]. Fixing $\mathbf{D}_{b}$, the optimization becomes a least square problem with quadratic constraints and can be treated by the Lagrange dual as used in [49].

\section{B. Structural Consistency Across Bands}

The goal of the SCSC method is to maintain the distinct band characteristics while maintaining a consistent structure across bands. While sounds contradictory, we get this done by different treatments to the dictionary learning and sparse coefficients estimation, which are two key components in sparse coding. To be more specific, different dictionaries are generated for 
different bands, so as to guarantee that the band-wise variation of spectral reflectance of materials can be accurately described. At the same time, we enforce the same coefficient in all bands when reconstructing a band image from the corresponding band specific dictionary.

Let $\mathbf{F}=\left[\mathbf{F}_{1}, \ldots, \mathbf{F}_{B}\right]$ be the set of data extracted from an HSI, where $B$ is the number of bands. $\mathbf{F}_{b}=\left[\mathbf{f}_{b 1}, \ldots, \mathbf{f}_{b M}\right] \in$ $\mathbb{R}^{N \times M}$ is the set of features extracted from the $b$ th band, where $M$ is the number of pixels and $N$ is the dimension of data. Note that the data at a pixel is extracted from a local neighborhood, either in the form of raw intensity values as in Section III-A, or certain image features such as discrete wavelet and so on. Then, we formulate the structural consistency model for the whole HSI as

$$
\begin{gathered}
\min _{\mathbf{D}_{1}, \ldots, \mathbf{D}_{B}, \mathbf{S}} \sum_{b=1}^{B}\left\|\mathbf{F}_{b}-\mathbf{D}_{b} \mathbf{S}\right\|^{2}+\beta\|\mathbf{S}\|_{1} \\
\text { s.t. }\left\|\mathbf{d}_{b i}\right\| \leq c, i=1, \ldots, R, b=1, \ldots, B
\end{gathered}
$$

where $\mathbf{D}_{1}, \ldots, \mathbf{D}_{B} \in \mathbb{R}^{N \times R}$ are the dictionaries for different bands, $R$ is the size of dictionary, and $\mathbf{S} \in \mathbb{R}^{R \times M}$ is the sparse coefficient matrix, which is the same for all bands.

\section{Extension With Graph Constraint}

In order to capture the structural relationship between the neighboring pixels and improve the discriminative capability of the learned model, sparse coding models are usually added with certain constraints [12], [36], [40]-[42]. In particular, recent work show that graph Laplacian sparsity constraint has demonstrated exceptional performance when compared with alternatives [12]. Graph Laplacian sparsity constraints can preserve the local manifold structure of data. If two data points $x_{i}$ and $x_{j}$ are close in their original space, the corresponding sparse coefficients $\mathbf{s}_{i}$ and $\mathbf{s}_{j}$ shall also be close in the new space.

Inspired by this idea, we also incorporate graph Laplacian sparsity constraint into the proposed method. This constraint builds the nearest neighbor graph using the spectral feature. This makes the learned sparse coefficients preserve the material spectral characteristics and the spatial correlations of pixels because local structures might be made of same materials.

Let the spectral features of a set of pixels be $\mathbf{X}=$ $\left[\mathbf{X}_{1}, \ldots, \mathbf{X}_{M}\right] \in \mathbb{R}^{B \times M}$, where $B$ is the number of bands, and $M$ is the number of pixels. We construct a K-nearest neighbor (KNN) graph $\mathbf{G}$ with $M$ vertices and calculate the weight matrix $\mathbf{W}$ of $\mathbf{G}$. If $\mathbf{X}_{i}$ is among the KNNs of $\mathbf{X}_{j}$ or $\mathbf{X}_{j}$ is among the KNNs of $\mathbf{X}_{i}, \mathbf{W}_{i j}=1$, otherwise, $\mathbf{W}_{i j}=0$. The Laplacian constraint is to map the weighted graph $\mathbf{G}$ to the sparse coefficients $\mathbf{S}$. It is defined as

$$
\operatorname{Tr}\left(\mathbf{S L S}^{T}\right)=\frac{1}{2} \sum_{i=1}^{M} \sum_{j=1}^{M}\left(\mathbf{s}_{i}-\mathbf{s}_{j}\right) \mathbf{W}_{i j}
$$

where $\mathbf{L}=\mathbf{P}-\mathbf{W}$ is the Laplacian matrix, $\mathbf{P}=\operatorname{diag}\left(p_{1}\right.$, $\left.\ldots, p_{M}\right)$ is a diagonal matrix and $p_{i}=\sum_{j=1}^{M} \mathbf{W}_{i j}$ is the degree of $\mathbf{X}_{i}$. Combining the Laplacian constraint into the sparse coding model in (3), the objective function of the graph-based sparse coding model with structural consistency is formulated as

$$
\begin{aligned}
& \underset{\mathbf{D}_{1}, \ldots, \mathbf{D}_{B}, \mathbf{S}}{\min _{b=1}} \sum_{\text {s.t. }}^{B}\left\|\mathbf{F}_{b i}\right\| \leq \mathbf{D}_{b} \mathbf{S}\left\|^{2}+\alpha \operatorname{Tr}\left(\mathbf{S} \mathbf{L} \mathbf{S}^{T}\right)+\beta\right\| \mathbf{S} \|_{1} \\
& \text { s }=1, \ldots, R, b=1, \ldots, B .
\end{aligned}
$$

\section{Iterative Optimization Process}

The sparse coding problem is usually solved by the $l_{1}$-norm minimization optimization methods [36], [49]. Followed by the $l_{1}$-norm minimization, the problem in (5) can be solved by alternatively optimizing the dictionaries and the sparse coefficients while fixing the other. More specifically, the optimization procedure includes two iterative steps: (1) learning sparse coefficients $\mathbf{S}$ while fixing the dictionaries $\mathbf{D}_{1}, \ldots, \mathbf{D}_{B}$, and (2) learning the dictionaries $\mathbf{D}_{1}, \ldots, \mathbf{D}_{B}$ while fixing the sparse coefficients $\mathbf{S}$.

1) Learning Sparse Coefficients $\mathbf{S}$ : When fixing the dictionaries $\mathbf{D}_{1}, \ldots, \mathbf{D}_{B}$, the sparse coefficients $\mathbf{S}$ learning problem becomes

$$
\min _{\mathbf{S}} \sum_{b=1}^{B}\left\|\mathbf{F}_{b}-\mathbf{D}_{b} \mathbf{S}\right\|^{2}+\alpha \operatorname{Tr}\left(\mathbf{S L S} \mathbf{S}^{T}\right)+\beta\|\mathbf{S}\|_{1} .
$$

This can be rewritten as

$$
\min _{\mathbf{S}} \sum_{i=1}^{M} \sum_{b=1}^{B}\left\|\mathbf{f}_{i b}-\mathbf{D}_{b} \mathbf{s}_{i}\right\|^{2}+\alpha \sum_{i, j=1}^{M} \mathbf{L}_{i j} \mathbf{s}_{i}^{T} \mathbf{s}_{j}+\beta \sum_{i=1}^{M}\left\|\mathbf{s}_{i}\right\|_{1}
$$

where $\left\|\mathbf{s}_{i}\right\|_{1}=\sum_{r=1}^{R}\left|\mathbf{s}_{i}^{(r)}\right|$ and $\mathbf{s}_{i}^{(r)}$ is the $r$ th element of $\mathbf{s}_{i}$. Each vector $\mathbf{S}_{i}$ in $\mathbf{S}$ can be updated individually while keeping all the others vectors $\left\{\mathbf{s}_{j}\right\}_{j \neq i}$ as constants. Then, the optimization problem for $\mathbf{s}_{i}$ becomes

$$
\begin{aligned}
\min _{\mathbf{s}_{i}} f\left(\mathbf{s}_{i}\right)= & \sum_{b=1}^{B}\left\|\mathbf{f}_{i b}-\mathbf{D}_{b} \mathbf{s}_{i}\right\|^{2}+\alpha \mathbf{L}_{i i} \mathbf{s}_{i}^{T} \mathbf{s}_{i} \\
& +\mathbf{s}_{i}^{T} \mathbf{h}_{i}+\beta\left\|\mathbf{s}_{i}\right\|_{1}
\end{aligned}
$$

where

$$
\mathbf{h}_{i}=2 \alpha\left(\sum_{j \neq i} \mathbf{L}_{i j} \mathbf{s}_{j}\right)
$$

Following the feature-sign search algorithm [49], the optimization problem in (8) can be implemented by searching for the optimal active set, which is a set of potentially nonzero coefficients, and their corresponding signs. The reasons are two-folds.

1) If we know the signs of all elements in $\mathbf{s}_{i}$, the optimization problem in (8) becomes a standard unconstrained quadratic optimization problem which can be solved analytically and efficiently.

2) The nonsmooth optimization theory [50] shows that the necessary condition for a parameter vector to be a local minima is that the zero-vector is an element of the subdifferential[36], so the active set $\hat{\mathbf{A}}$ can be obtained by

$$
\hat{\mathbf{A}}=\left\{j\left|\mathbf{s}_{i}^{(j)}=0,\right| \nabla_{i}^{(j)} g_{s}\left(\mathbf{s}_{i}\right) \mid>\beta\right\}
$$

where $\nabla_{i}^{(j)}$ denotes the subdifferentiable value of the $j$ th element of $\mathbf{s}_{i}, \mathbf{s}_{i}^{(j)}$ is the $j$ th element of $\mathbf{s}_{i}$ and 
$g_{s}\left(\mathbf{s}_{i}\right)=\sum_{b=1}^{B}\left\|\mathbf{f}_{i b}-\mathbf{D}_{b} \mathbf{s}_{i}\right\|^{2}+\alpha \mathbf{L}_{i i} \mathbf{s}_{i}^{T} \mathbf{s}_{i}+\mathbf{s}_{i}^{T} \mathbf{h}_{i}$. For each iteration, the $j$ th element with the largest subgradient value is selected into the active set from zero-value elements of $\mathbf{s}_{i}$ given by

$$
j=\arg \max _{j}\left|\nabla_{i}^{(j)} g_{s}\left(\mathbf{s}_{i}\right)\right| .
$$

To locally improve this objective, the $\operatorname{sign} \theta_{j}$ of $\mathbf{s}_{i}^{(j)}$ is estimated by

$$
\theta_{j}= \begin{cases}-1, & \text { if } \nabla_{i}^{(j)} g_{s}\left(\mathbf{s}_{i}\right)>\beta \\ 1, & \text { if } \nabla_{i}^{(j)} g_{s}\left(\mathbf{s}_{i}\right)<-\beta .\end{cases}
$$

Let $\hat{\theta}$ be the signs corresponding to the active set $\hat{\mathbf{A}}$. The optimization problem in (8) reduces to a standard unconstrained quadratic optimization problem

$$
\min _{\hat{\mathbf{s}}_{i}} f_{\text {new }}\left(\hat{\mathbf{s}}_{i}\right)=\sum_{b=1}^{B}\left\|\mathbf{f}_{i b}-\hat{\mathbf{D}}_{b} \hat{\mathbf{s}}_{i}\right\|^{2}+\alpha \mathbf{L}_{i i} \hat{\mathbf{s}}_{i}^{T} \hat{\mathbf{s}}_{i}+\hat{\mathbf{s}}_{i}^{T} \hat{\mathbf{h}}_{i}+\beta \hat{\theta} .
$$

This can be solved efficiently and the optimal value of $\mathbf{s}_{i}$ over the current active set can be obtained by letting $\left(\partial f_{\text {new }}\left(\hat{\mathbf{s}}_{i}\right) / \partial \hat{\mathbf{s}}_{i}\right)=$ 0 . Then we get

$\hat{\mathbf{s}}_{i}^{\text {new }}=\left(\sum_{b=1}^{B} \hat{\mathbf{D}}_{b}^{T} \hat{\mathbf{D}}_{b}+\alpha \mathbf{L}_{i i} \mathbf{I}\right)^{-1}\left(\sum_{b=1}^{B} \hat{\mathbf{D}}_{b}^{T} \mathbf{f}_{b i}-\left(\hat{\mathbf{h}}_{i}+\beta \hat{\theta}\right) / 2\right)$

where $\mathbf{I}$ is the identity matrix. Then, the line search is performed between the current solution $\hat{\mathbf{s}}_{i}$ and $\hat{\mathbf{s}}_{i}^{\text {new }}$ to search for the optimal active set and signs which can minimize the objective function in (8) and get the optimal solution mathb $f s_{i}^{*}$. The algorithm for learning the sparse coefficients $\mathbf{S}$ is summarized in Algorithm 1.

2) Learning Dictionaries $\mathbf{D}_{1}, \ldots, \mathbf{D}_{B}$ : In our method, different dictionaries are generated for different bands, but these bands share the same sparse coefficients $\mathbf{S}$ for preserving consistent structures in the image. The $b$ th dictionary is constructed from data $\mathbf{F}_{b}$ in the $b$ th band, so the dictionary in each band can be learned individually when fixing the sparse coefficients $\mathbf{S}$. The problem of learning the dictionary becomes a least squares problem with quadratic constraints for each band, so the dictionaries $\mathbf{D}_{1}, \ldots, \mathbf{D}_{B}$ can be obtained separately by the following objective function:

$$
\begin{gathered}
\min _{\mathbf{D}_{1}}\left\|\mathbf{F}_{1}-\mathbf{D}_{1} \mathbf{S}\right\|_{2} \\
\min _{\mathbf{D}_{2}}\left\|\mathbf{F}_{2}-\mathbf{D}_{2} \mathbf{S}\right\|_{2} \\
\vdots \\
\min _{\mathbf{D}_{B}}\left\|\mathbf{F}_{B}-\mathbf{D}_{B} \mathbf{S}\right\|_{2} .
\end{gathered}
$$

This optimization problem can be solved by the Lagrangian dual method. The whole procedure is summarized in Algorithm 2.

\section{E. Discussion and Analysis}

To get better understanding of the learned dictionaries and coefficients, we analyze the outcome of the proposed method on the Indian Pines dataset. In this experiment, the size of dictionary
Algorithm 1: Learning Sparse Coefficients S Based on

Feature-Sign Search.

\section{Input:}

Data from $B$ bands $\mathbf{F}_{1}, \ldots, \mathbf{F}_{B} \in \mathbb{R}^{N \times M}$.

Dictionaries from $B$ bands $\mathbf{D}_{1}, \ldots, \mathbf{D}_{B} \in \mathbb{R}^{N \times R}$.

Laplacian matrix $\mathbf{L}$, regularization parameters $\beta$ and $\alpha$.

\section{Output:}

The optimal coefficient matrix $\mathbf{S}=\left[\mathbf{s}_{1}^{*}, \ldots, \mathbf{s}_{M}^{*}\right]$.

1: for each $i \in[1, M]$ do

2: initialize :

3: $\quad \mathbf{s}_{i}=\overrightarrow{0}, \theta=\overrightarrow{0}$ and the active set $\mathbf{A}=\varnothing$.

4: activate :

5: $\quad$ Select $j$ using Eq. (11), activate $\mathbf{s}_{i}^{(j)}(\mathbf{A}=\mathbf{A} \cup\{j\})$ and update the sign $\theta_{j}$ of $\mathbf{s}_{i}^{(j)}$ using Eq. (12).

6: feature - sign :

7: $\quad$ Let $\hat{\mathbf{D}}_{1}, \ldots, \hat{\mathbf{D}}_{B}$ be the sub-matrices of $\mathbf{D}_{1}, \ldots, \mathbf{D}_{B}$ that contain only the columns corresponding to the active set.

8: $\quad$ Let $\hat{\mathbf{s}}_{i}, \hat{\theta}$, and $\hat{\mathbf{h}}_{i}$ be the sub-vectors of $\mathbf{s}_{i}, \theta$, and $\mathbf{h}_{i}$ corresponding to the active set.

9: $\quad$ Solve the unconstrained quadratic optimization problem using (13) and get the optimal value of $\mathbf{s}_{i}$ over the current active set using (14).

10: Perform a discrete line search on the closed line segment from $\hat{\mathbf{s}}_{i}$ to $\hat{\mathbf{s}}_{i}^{\text {new }}$ and update $\hat{\mathbf{s}}_{i}$ to the point with the lowest objective value.

11: Remove the zero value of $\hat{\mathbf{s}}_{i}$ from the active set and update $\theta=\operatorname{sign}\left(\mathbf{s}_{i}\right)$.

12: check the convergence conditions:

13: (a) Convergence condition for nonzero coefficients: $\nabla_{i}^{(j)} g_{s}\left(\mathbf{s}_{i}\right)+\beta \operatorname{sign}\left(\mathbf{s}_{i}^{(j)}\right)=0, \forall \mathbf{s}_{i}^{(j)} \neq 0$. If condition

(a) is not satisfied, go to Step 6. Otherwise check condition (b).

14: (b) Covergence condition for zero coefficients: $\left|\nabla_{i}^{(j)} g_{s}\left(\mathbf{s}_{i}\right)\right| \leq \beta$. If condition (b) is not satisfied, go to Step 4 , otherwise return $\mathbf{s}_{i}$ as the optimal solution $\mathbf{s}_{i}^{*}$.

15: end for

is set to 30 . Image patches of size $9 \times 9$ centered at each pixel are used as the input. Fig. 3 shows the obtained dictionaries from the 70th to the 100th bands, in which each cell represents a base vector in a dictionary and each row shows the dictionary learned from a band.

Two significant characteristics can be observed from this figure. First, the dictionaries have great similarities across bands, which reflects the inherent structural consistency across bands and also validates the effectiveness of the proposed method. Second, there are slight changes across bands, thanks to the band specific dictionaries. These two characteristics verify that the learned dictionaries can preserve the structural information in the spectral responses, while depicting their fine differences.

Based on the learned dictionaries, we select six pixels to compute their sparse coefficients. The location of these pixels are displayed in Fig. 4. The corresponding sparse coefficients 

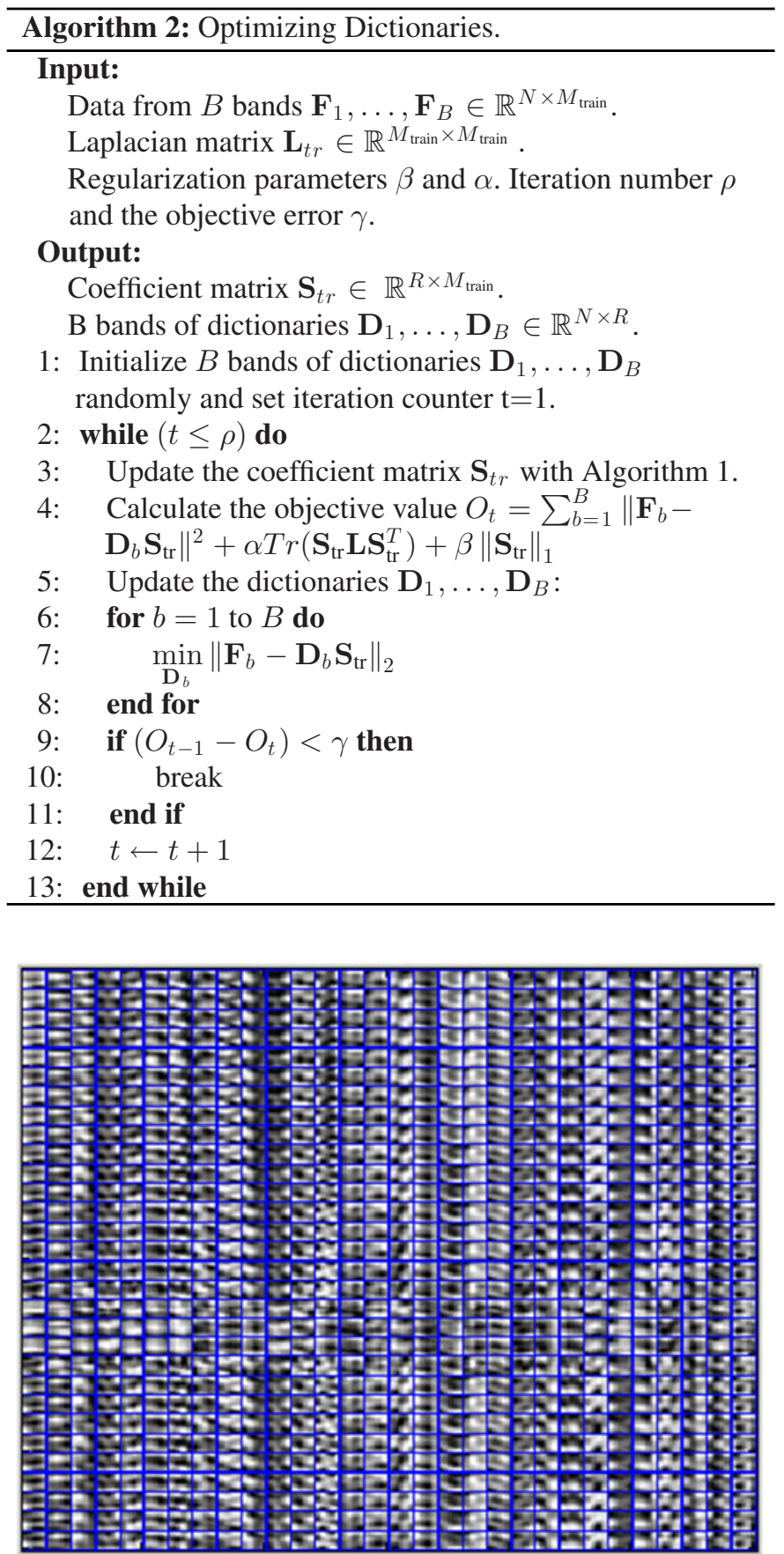

Fig. 3. Learned dictionaries from band 70 to band 100 in the Indian Pines dataset. Each row represents a dictionary learned from a band, including 30 base vectors.

are shown in Fig. 5. From Fig. 5, we can observe that the same class of pixels have very similar sparse coefficients and different classes of pixels have different sparse coefficients. Local patch of pixel 4 is constructed from two classes, so its sparse coefficient is a mixture of those from pixels 1 and 2 and is similar partly to that of pixel 5 . The phenomenon maybe the result from the structural consistency in the proposed method.

From the above analysis, we can see that the proposed method can indeed capture the structural consistency across bands and spectral responses in different bands.

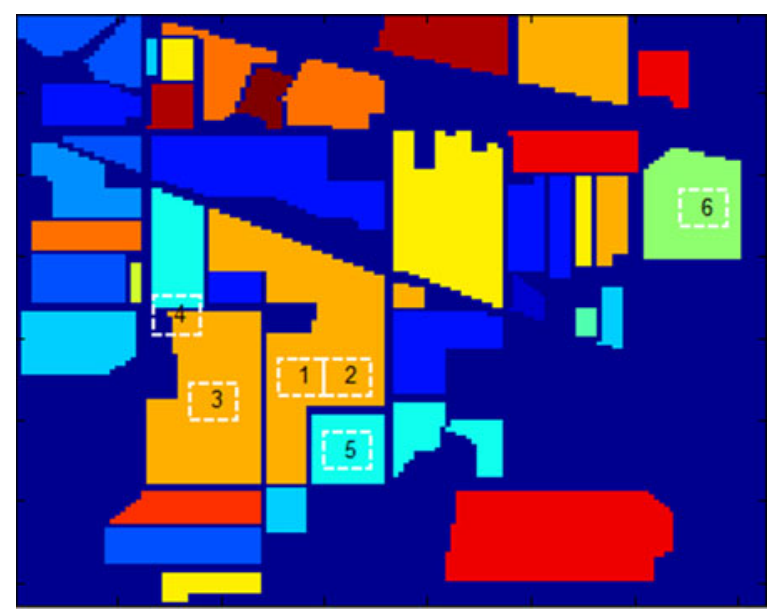

Fig. 4. Locations of six pixels and corresponding patches in the Indian Pines dataset for coefficients calculation. Pixels 1-4 are from "Soybean-mintill" class. Pixels 5 and 6 are from "Grass-trees" and "Hay-windrowed" classes, respectively.

\section{EXPERIMENTS}

In this section, we demonstrate the effectiveness of the proposed approach on two benchmark hyperspectral remote sensing datasets ${ }^{1}$ : Indian Pines dataset captured by AVIRIS (Airborne Visible/Infrared Imaging Spectrometer) and University of Pavia dataset captured by ROSIS (Reflective Optics System Imaging Spectrometer). To validate the proposed SCSC method and its graph regularized extension (GSCSC) method, we compare them with the following methods:

1) SCS: a baseline sparse coding method built on only spectral feature.

2) SC-Single: patch-based sparse coding built on only one band, without using structural consistency. In this method, only the coefficients of a clear band are used for classification.

3) SCNSC: sparse coding without considering structural consistency built on all bands. Sparse coding is run on each band respectively, and then the sparse coefficients calculated on all bands are concatenated into a vector as the input to the SVM classifier.

4) Two state-of-the-art spectral-spatial sparse coding methods, including SOMP [44] and SWSC [48].

In SC-Single, SCNSC, SCSC, and GSCSC, the grayscale values in a image patch are used as the 2-D feature. Both SOMP and SWSC use the spectral responses as the features. For fair comparison, we did not adopt the spatially smoothed version of SWSC. Additionally, we also analyze the influence of several model parameters, including patch size, dictionary size, and number of training samples.

\section{A. Hyperspectral Datasets}

The Indian Pines image was acquired by AVIRIS sensor over the Indian Pines test site in North western Indiana in 1992 [51].

\footnotetext{
${ }^{1}$ http://www.ehu.eus/ccwintco/index.php?title=Hyperspectral_Remote_Sen sing_Scenes
} 

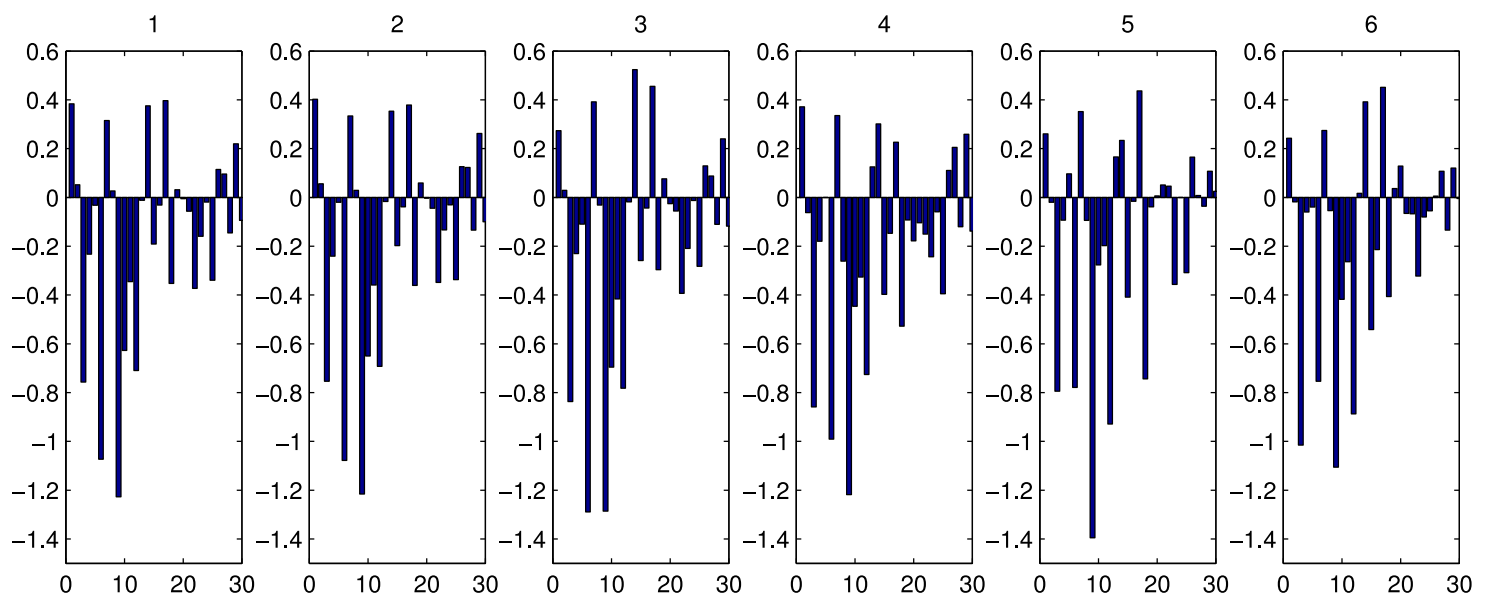

Fig. 5. Sparse coefficients of six pixels in Fig. 4 .

It consists of 220 spectral bands in the wavelength range from 0.4 to $2.5 \mu \mathrm{m}$ and the image size is $145 \times 145$ for each band. 200 bands are used for experiments after 20 noisy bands (bands 104-108, 150-163, and 220) are removed [7]. The HSI contains 16 classes of land-covers including 10366 labeled pixels.

The University of Pavia dataset was acquired by the ROSIS sensor during a flight campaign over the University of Pavia, northern Italy. The number of spectral bands is 103 after removing some noisy bands, and the size of each band image is $610 \times 340$. There are nine land-cover classes in total.

\section{B. Experimental Setting}

Unless otherwise specified, the parameters are set as follows in the experiments for the proposed method. The regularization parameter $\beta$ in (3) and (5) is fixed to 0.1. $\alpha$ in (5) is fixed to 1.0. $K$ in $\mathrm{KNN}$ graph constraint is set to 3 . The size of dictionary is set to 30 .

For each image, we first solve the sparse coefficients for SCS, SC-Single, SCNSC, SCSC, and GSCSC.gg Then, the solved sparse coefficients are fed into the nonlinear SVMs with an RBF kernel [52] for classification, except for SCNSC, which uses linear SVM due to is high-dimensional feature. The RBF-kernel parameters in SVM are obtained by cross-validation. The sparse coefficients are solved by the $\ell_{1}$ optimization algorithms [49] for SCS, SC-Single, and SCNSC. The parameter settings of SOMP and SWSC are followed by the implements of Farani and Rabiee ${ }^{2}$ with $K_{0}=30, \lambda=150, \alpha=800$, and $\beta=800$.

In GSCSC, we first construct the KNN graph matrix $\mathbf{W}$ with spectral features from randomly selected training samples $\mathbf{X}$ and estimate the dictionaries in an unsupervised manner. This dictionary is then used in the testing stage to calculate the sparse coefficient of a new data $x_{i}$. Here, the graph matrix $\mathbf{W}$ is modified as $\left[\begin{array}{cc}\mathbf{W} & \mathbf{w}_{i} \\ \mathbf{w}^{T} & 0\end{array}\right]$, where $\mathbf{w}_{i}$ is a weight vector of KNNs extracted from $x_{i}$ in $\mathbf{X}$. The classification performance is evaluated by overall accuracy (OA), average accuracy (AA), and $\kappa$ coefficient measure [53]. OA is the percentage of correctly classified

\footnotetext{
${ }^{2}$ Codes of SOMP and SWSC were extracted from http://ssp.dml.ir/research/swsc/
}

samples among all testing samples. AA is the mean of the classspecific accuracies. $\kappa$ coefficient measures the degree of agreement in classification.

\section{Influence of Model parameters}

In the GSCSC model, there are tree key parameters: the sparsity regularization parameter $\beta$, the Laplacian constraint parameter $\alpha$, and $K$ in KNN graph constraint. We test their influence to the classification performance on the Indian Pines dataset. The average results of five-runs on $10 \%$ randomly sampled training set are reported for all methods. Because only GSCSC has the Laplacian constraint parameter $\alpha$ and $K$ in KNN graph constraint, we analyze the influence of $\alpha$ and $K$ to GSCSC only. The range of $\beta$ is $\{0.005,0.01,0.03,0.05,0.08,0.1,0.3,0.5$, $0.8\}$, the range of $\alpha$ is $\{0.01,0.1,1.0,10,100\}$, and the range of $K$ is $\{2,3,4,5,6,7,8,9,10\}$, respectively.

The classification performance of different $\beta$ values are shown in Fig. 6(a). The results show that models with no structural consistency are greatly influenced by the $\beta$ value. Their performance fall abruptly when $\beta$ is greater than 0.1 . This is because the learned sparse coefficients will be too sparse and most entries are close to zero for SCS, SC-Single, and SCNSC. SCS performs better when $\beta$ is small. SC-Single and SCNSC work the best at 0.1 . Models with structural consistency are much less influenced by small $\beta$. GSCSC changes more smoothly than SCSC. This implies that the graph regularized term smooths the model with spectral information and reduces the importance of the sparsity.

The classification performance of different $\alpha$ values are shown in Fig. 6(b). This figure shows that the performance of GSCSC decreases when $\alpha$ value is too large. In the proposed model, $\alpha$ controls the contribution from the graph regularized term. When it is too large, the influence from structural consistency and sparsity are suppressed. Therefore, it is preferable to set $\alpha$ value between 1.0 and 10 for GSCSC.

The classification performance of different $K$ in KNN graph constraint are shown in Fig. 6(c). GSCSC achieved very good performance with $3 \mathrm{NN}$ and $9 \mathrm{NN}$, however, $3 \mathrm{NN}$ requires much 


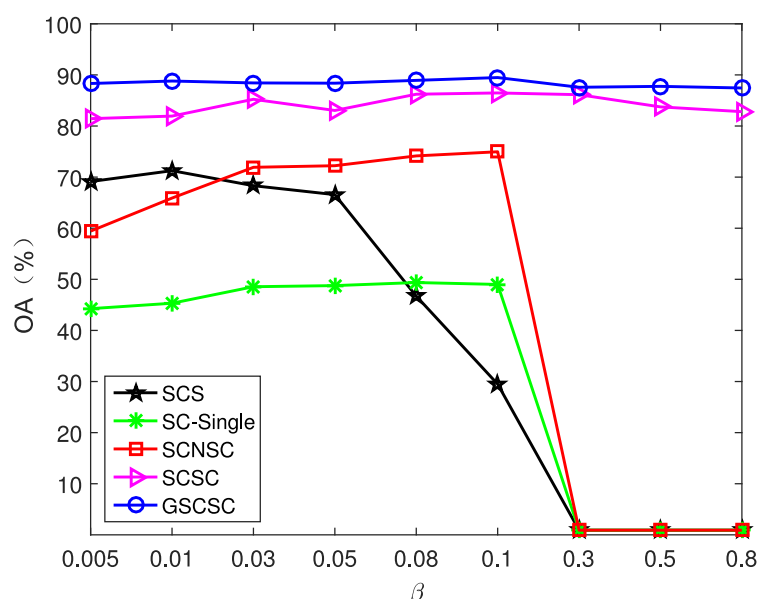

(a)

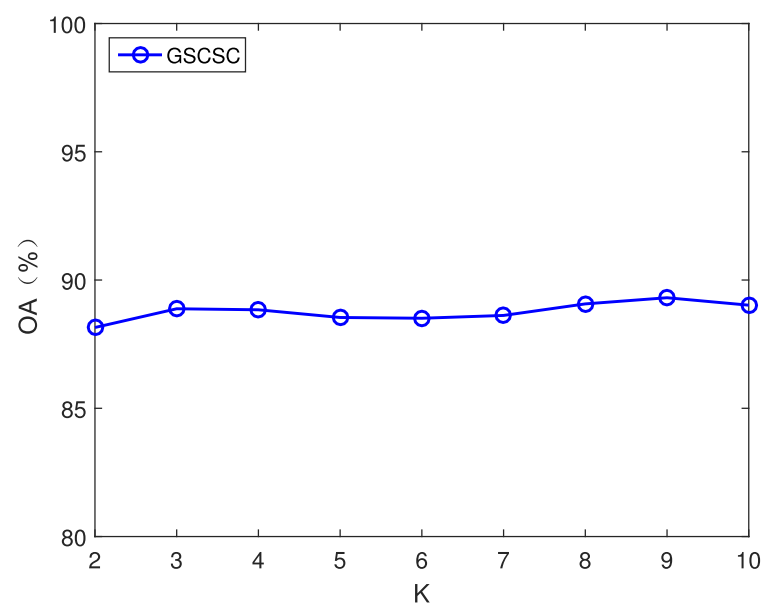

(c)

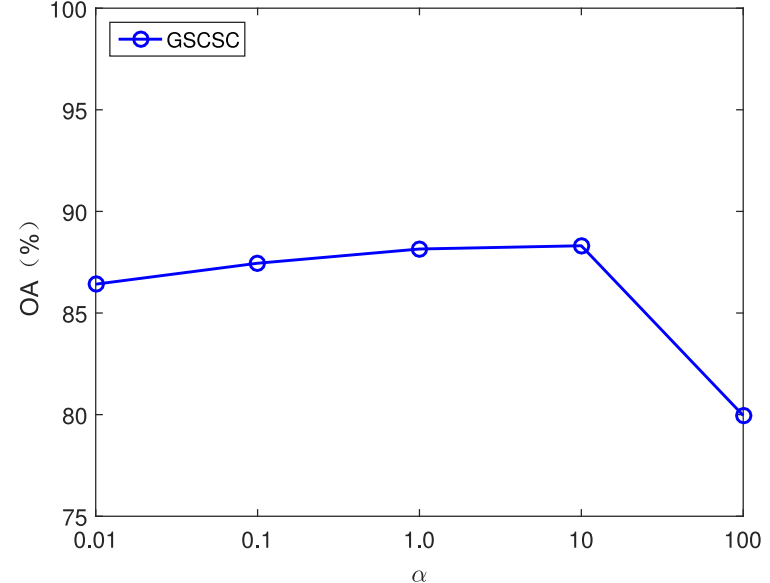

(b)

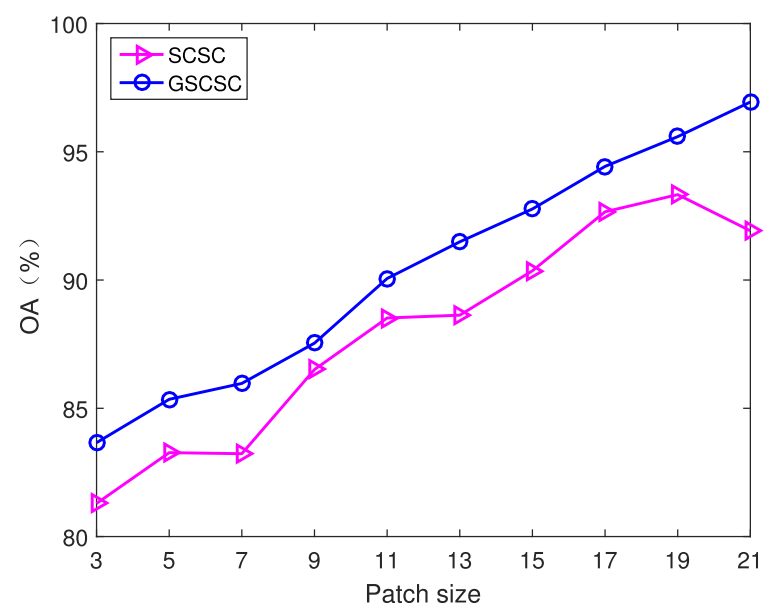

(d)

Fig. 6. Classification performance under different (a) $\beta$ values, (b) $\alpha$ values, (c) $K$ in KNN graph constraint, and (d) patch sizes.

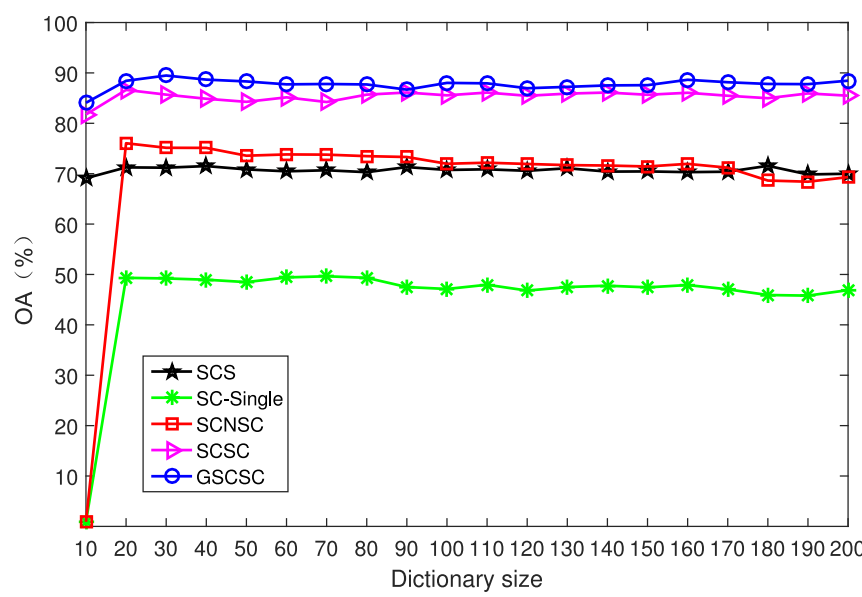

Fig. 7. Classification performance under different dictionary sizes.

less computation than 9NN. Therefore, in the rest of the experiments, we set $K=3$.

\section{Influence of Patch Size}

It is well known that the patch size have an impact on classification of HSI. We analyze the classification performance with
TABLE I

16 Classes in the Indian Pines IMAGe AND TRAInING/Testing SETS FOR EACH Class

\begin{tabular}{|c|c|c|c|}
\hline \multirow[b]{2}{*}{ No. } & \multirow{2}{*}{$\begin{array}{c}\text { Class } \\
\text { Class name }\end{array}$} & \multicolumn{2}{|c|}{ Samples } \\
\hline & & Training & Testing \\
\hline 1 & Alfalfa & 5 & 41 \\
\hline 2 & Corn-notill & 143 & 1285 \\
\hline 3 & Corn-mintill & 83 & 747 \\
\hline 4 & Corn & 24 & 213 \\
\hline 5 & Grass-pasture & 48 & 435 \\
\hline 6 & Grass-trees & 73 & 657 \\
\hline 7 & Grass-pasture-mowed & 3 & 25 \\
\hline 8 & Hay-windrowed & 48 & 430 \\
\hline 9 & Oats & 2 & 18 \\
\hline 10 & Soybean-notill & 97 & 875 \\
\hline 11 & Soybean-mintill & 246 & 2209 \\
\hline 12 & Soybean-clean & 59 & 534 \\
\hline 13 & Wheat & 21 & 184 \\
\hline 14 & Woods & 127 & 1138 \\
\hline 15 & Buildings-Grass-Trees-Drives & 39 & 347 \\
\hline \multirow[t]{2}{*}{16} & Stone-Steel-Towers & 9 & 84 \\
\hline & Total & 1027 & 9222 \\
\hline
\end{tabular}

different patch sizes $\{3 \times 3,5 \times 5,7 \times 7,9 \times 9,11 \times 11,13 \times$ $13,15 \times 15,17 \times 17,19 \times 19,21 \times 21\}$. Fig. $6(d)$ shows that the performance of both SCSC and GSCSC increases with larger 


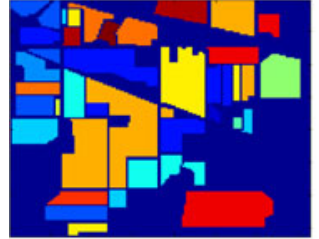

(a)

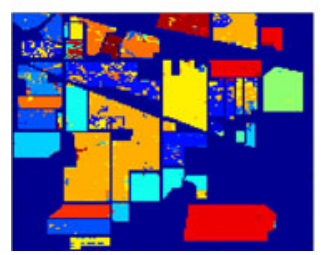

(f)

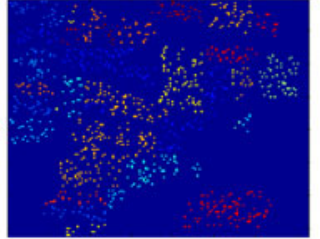

(b)

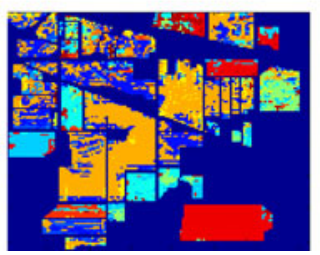

(g)

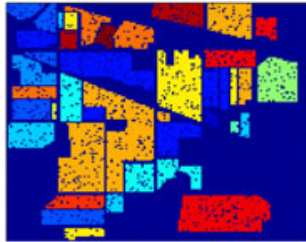

(c)

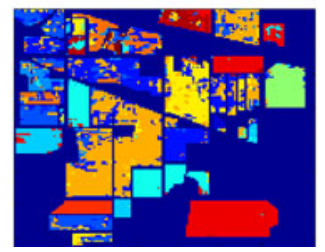

(h)

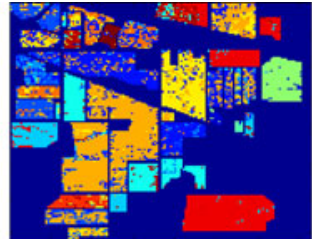

(d)

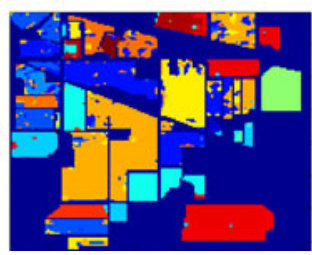

(i)

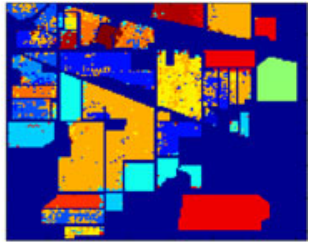

(e)

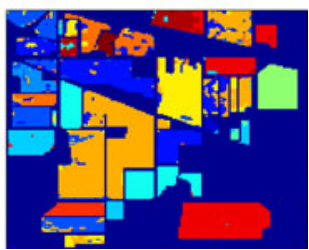

(j)

Fig. 8. Results on the Indian Pines dataset. (a) ground truth, (b) training set, and (c) testing set. Classification maps obtained by (d) SCS, (e) SOMP [44], (f) SWSC [48], (g) SC-Single, (h) SCNSC, (i) SCSC, and (j) GSCSC.

TABLE II

Nine Classes in the University of PAVIa Dataset AND Training/Testing SETS FOR EACH CLASS

\begin{tabular}{llcc}
\hline \hline & \multicolumn{2}{c}{ Class } & \multicolumn{2}{c}{ Samples } \\
\cline { 3 - 4 } No. & \multicolumn{1}{c}{ Class name } & Training & Testing \\
\hline 1 & Asphalt & 597 & 6034 \\
2 & Meadows & 1678 & 16971 \\
3 & Gravel & 189 & 1910 \\
4 & Trees & 276 & 2788 \\
5 & Painted metal sheets & 121 & 1224 \\
6 & Bare Soil & 453 & 4576 \\
7 & Bitumen & 120 & 1210 \\
8 & Self-Blocking Bricks & 331 & 3351 \\
9 & Shadows & 85 & 862 \\
& Total & 3850 & 38926 \\
\hline \hline
\end{tabular}

patch size. However, the performance of SCSC starts to drop when the patch size reaches 21 . This suggests that an appropriate patch size shall be set to obtain appropriate spatial distribution in a local neighborhood. For fair comparison with other methods, we follow the same setting in SOMP [44] and use $9 \times 9$ patch on the Indian Pines dataset and $5 \times 5$ patch on the University of Pavia dataset.

\section{E. Influence of Dictionary Size}

The sparse coding models are usually influenced easily by the dictionary size, so we look into this factor on the Indian pines dataset. We carry out the experiments with dictionary size $\{10$, 20, 30, 40, 50, 60, 70, 80, 90, 100, 110, 120, 130, 140, 150, 160, $170,180,190,200\}$ and report the average results of five-runs with $10 \%$ randomly sampled training set. Other experimental setup is the same as that of Section IV-B.

Fig. 7 shows the classification performance of the methods with different dictionary sizes. Both SCSC and GSCSC achieve $85 \%$ accuracy when the dictionary size reaches 20 , then their performance do not change much with larger dictionary. Fig. 7 indicates that our methods can learn the discriminative sparse coefficients and achieve good performance with small dictionary size. SCSC and GSCSC obtain better results than SC-Single and
TABLE III

Classification ACCURACIES (\%) ON INDIAN PINES DATASET With 10\% LABELED SAMPLES USED FOR TRAINING

\begin{tabular}{lccccccc}
\hline \hline Class & SCS & SOMP $[44]$ & SWSC $[48]$ & SC-Single & SCNSC & SCSC & GSCSC \\
\hline 1 & 80.49 & 75.61 & $\mathbf{9 5 . 1 2}$ & 43.90 & 85.37 & 85.37 & 90.24 \\
2 & 65.29 & 73.46 & $\mathbf{8 8 . 7 2}$ & 42.65 & 70.82 & 84.67 & 84.12 \\
3 & 51.94 & 59.04 & $\mathbf{9 5 . 4 5}$ & 20.48 & 68.14 & 83.40 & 85.27 \\
4 & 31.46 & 59.15 & $\mathbf{7 5 . 1 2}$ & 12.68 & 53.05 & 52.58 & $\mathbf{7 5 . 1 2}$ \\
5 & 81.61 & 94.02 & 93.33 & 67.82 & 83.22 & 94.94 & $\mathbf{9 6 . 0 9}$ \\
6 & 88.89 & 97.56 & 95.59 & 58.14 & 94.52 & 96.35 & $\mathbf{9 8 . 7 8}$ \\
7 & 80.00 & 44.00 & $\mathbf{9 6 . 0 0}$ & 20.00 & 32.00 & 68.00 & 80.00 \\
8 & 93.26 & $\mathbf{1 0 0 . 0 0}$ & 97.21 & 53.26 & 92.09 & 99.30 & $\mathbf{1 0 0 . 0 0}$ \\
9 & 11.11 & 22.22 & $\mathbf{5 5 . 5 6}$ & 44.44 & 27.78 & 38.89 & 38.89 \\
10 & 61.94 & 60.34 & $\mathbf{9 2 . 9 1}$ & 9.94 & 60.57 & 79.66 & 79.66 \\
11 & 74.06 & $\mathbf{9 3 . 0 3}$ & $\mathbf{6 8 . 9 9}$ & 69.71 & 73.83 & 86.69 & 88.82 \\
12 & 41.01 & 58.24 & $\mathbf{8 7 . 4 5}$ & 9.18 & 49.81 & 74.91 & 76.59 \\
13 & 75.00 & 92.93 & 99.46 & 65.76 & $\mathbf{1 0 0 . 0 0}$ & 95.65 & 99.46 \\
14 & 95.87 & $\mathbf{9 9 . 9 1}$ & 96.84 & 89.10 & 95.96 & 97.80 & 97.63 \\
15 & 44.09 & 81.27 & $\mathbf{8 8 . 7 6}$ & 34.29 & 60.23 & 70.32 & 77.23 \\
16 & 91.67 & $\mathbf{9 8 . 8 1}$ & $\mathbf{9 8 . 8 1}$ & 11.90 & 35.71 & 79.76 & 94.05 \\
OA & 70.97 & 82.45 & 86.94 & 49.93 & 74.83 & 86.39 & $\mathbf{8 8 . 3 5}$ \\
AA & 66.73 & 75.60 & $\mathbf{8 9 . 0 8}$ & 40.83 & 67.69 & 80.52 & 85.12 \\
$\kappa$ & 0.678 & 0.797 & 0.853 & 0.417 & 0.712 & 0.845 & $\mathbf{0 . 8 6 7}$ \\
\hline \hline & & & & & & & \\
\hline
\end{tabular}

SCNSC. This is because the proposed method explores structural consistency for better image description, but the alternatives do not have this property.

\section{F. Results on the Indian Pines Dataset}

On the Indian Pines dataset, we follow the same experiment settings to generate the training sets, the testing sets, and the patch size as SOMP [44]. Around $10 \%$ of the labeled samples are randomly chosen for training the classifier and the remaining are used for testing, as shown in Table I and Fig. 8(b) and (c), respectively. In the Indian Pines image, a large patch size $9 \times 9$ is used for all methods except for SCS. The Indian Pines image contains a lot of noises so that SCNSC gets very bad results in some bands when the sparse coefficients are calculated band by band separately. So we select some high-quality bands to calculate the concatenated vectors of SCNSC, e.g., bands 6-52. For SC-Single, we use only the 30th band, which is a highquality band. 


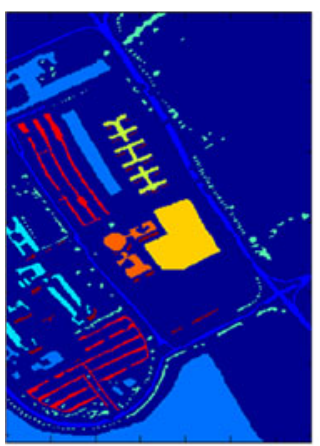

(a)

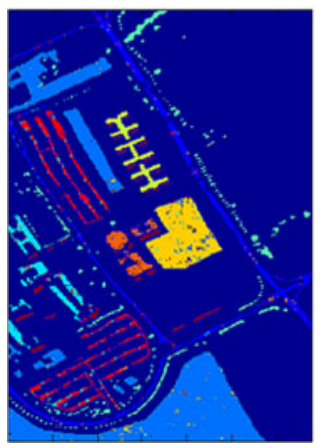

(f)

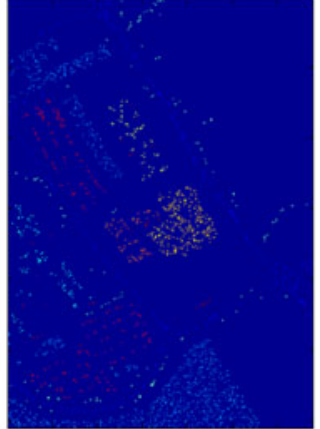

(b)

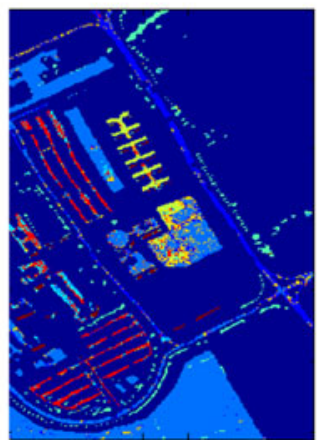

(g)

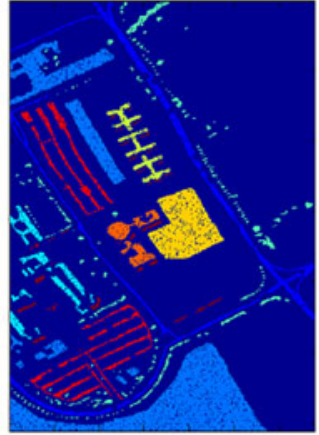

(c)

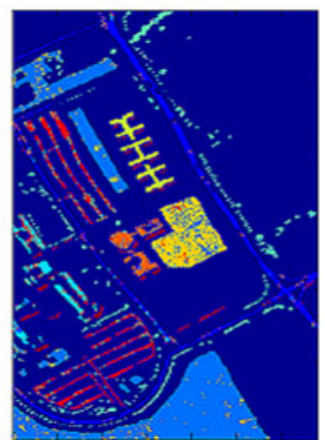

(h)

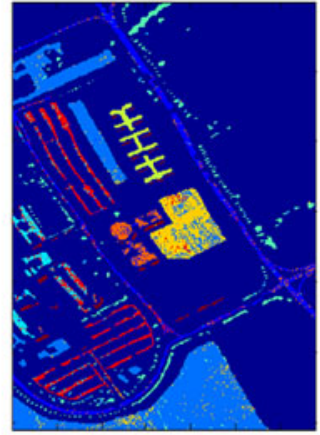

(d)

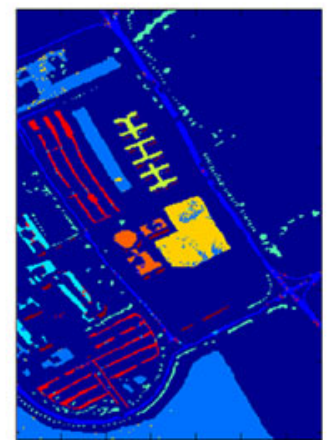

(i)

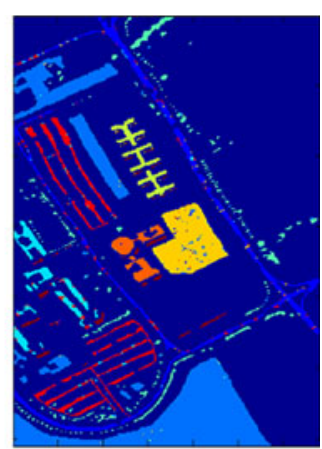

(e)

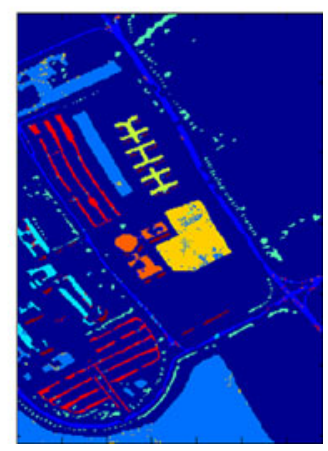

(j)

Fig. 9. Results on the University of Pavia dataset. (a) ground truth, (b) training set, and (c) testing set. Classification maps obtained by (d) SCS, (e) SOMP [44], (f) SWSC [48], (g) SC-Single, (h) SCNSC, (i) SCSC, and (j) GSCSC.

The classification results of the proposed methods and alternatives are shown in Table III. It can be seen that SCSC and GSCSC, which use structural consistency in the modeling, have significantly improved the classification accuracy over the baseline sparse coding method SCS. SC-Single in one band generates very bad result. This is because SC-Single only contains spatial information in a band and no any spectral information. SCNSC generates lower performance than SCSC and GSCSC since SCSC and GSCSC are benefit from structural consistency. Raw image data may contain large amount of noises so that the structures in every band of image are less clear and the structures centered on some pixels are easily get mixed with noises. This makes SCNSC difficult to generate good performance. This result validates the effectiveness of our proposed methods with structural consistency. Moreover, GSCSC which is a model with the graph Laplacian sparsity constraints, has shown better classification performance than the corresponding nongraph regularized SCSC. Compared with SOMP and SWSC, our method GSCSC has obtained better result.

The classification maps on labeled pixels are presented in Fig. 8(i) and (j). From this figure, we can see that the proposed methods can effectively capture the inherent consistent structures. Many pixels at the interior regions are misclassified by the SCS, SC-Single, and SCNSC methods. On the contrary, most errors happen at the boundary regions in the results generated by SCSC and GSCSC methods. This implies that the local spatial structures are similar in different bands at the internal regions of every class. At the boundary pixels, the structure information of neighboring class may get involved, which causes the errors because the learned consistent structures are easily confused between two neighboring classes. SOMP and SWSC with local spatial correlations also achieve better performance at big regions as shown in Fig. 8(e) and (f).

\section{G. Results on the University of Pavia Dataset}

We use the same experimental settings as in [44] to generate the training set, the testing set, and the patch size on the University of Pavia dataset. Around $9 \%$ of the labeled samples are randomly chosen for training and the remaining are used for testing. More detailed information on the training and testing sets are shown in Table II and Fig. 9(b) and (c). Considering the small spatial homogeneity in the University of Pavia image, a small patch size of $5 \times 5$ is used in all methods except SCS. SCNSC uses all bands of sparse coefficients and concatenates them into a vector.

The classification results of various methods are presented in Table IV. Fig. 9(d)-(j) gives the classification maps on labeled pixels. The proposed methods achieve great improvement over the baseline SCS and are better than SC-Single and SCNSC with no structural consistency. They also generate slightly higher performance than SOMP and SWSC, which are based on spatial correlations. From Fig. 9, we can see that the "Meadows" class at the bottom of the HSI is a large region and its internal pixels should have similar spatial structure and spectral information. Therefore, most pixels in this class are classified correctly by our methods. Because SCS captures only spectral information and SCNSC uses only spatial information, they are strongly influenced by the noises in different bands. The proposed methods 
TABLE IV

CLASSIFICATION ACCURACIES (\%) ON UNIVERSITY OF PAVIA DATASET WITH 9\% LABELED SAMPLES USED FOR TRAINING.

\begin{tabular}{lccccccc}
\hline \hline Class & SCS & SOMP [44] & SWSC [48] & SC-Single & SCNSC & SCSC & GSCSC \\
\hline 1 & 87.12 & 90.88 & 95.13 & 61.37 & 94.35 & $\mathbf{9 6 . 5 4}$ & 96.22 \\
2 & 95.24 & $\mathbf{9 9 . 7 3}$ & 98.06 & 96.03 & 95.35 & 98.06 & 98.83 \\
3 & 62.36 & 90.05 & 85.34 & 34.76 & 71.78 & 89.79 & $\mathbf{9 0 . 1 0}$ \\
4 & 88.56 & 91.50 & 97.53 & 90.85 & 96.70 & 97.85 & $\mathbf{9 7 . 9 9}$ \\
5 & 99.75 & $\mathbf{1 0 0 . 0 0}$ & 99.18 & 81.62 & 99.75 & 99.02 & $\mathbf{1 0 0 . 0 0}$ \\
6 & 72.14 & $\mathbf{9 4 . 8 4}$ & 89.82 & 21.92 & 77.45 & 87.02 & 88.35 \\
7 & 72.07 & $\mathbf{9 7 . 5 2}$ & 72.23 & 1.32 & 76.94 & 87.52 & 91.07 \\
8 & 83.97 & 95.26 & 75.44 & 76.54 & 81.65 & 94.63 & $\mathbf{9 5 . 6 4}$ \\
9 & 97.91 & 93.50 & $\mathbf{9 9 . 6 5}$ & 84.34 & $\mathbf{9 9 . 6 5}$ & 94.08 & 97.22 \\
OA & 87.68 & 96.16 & 93.29 & 73.24 & 90.51 & 95.42 & $\mathbf{9 6 . 1 9}$ \\
AA & 84.35 & 94.86 & 90.26 & 60.97 & 88.18 & 93.38 & $\mathbf{9 5 . 0 5}$ \\
$\kappa$ & 0.836 & $\mathbf{0 . 9 4 9}$ & 0.911 & 0.627 & 0.874 & 0.939 & $\mathbf{0 . 9 4 9}$ \\
\hline \hline
\end{tabular}

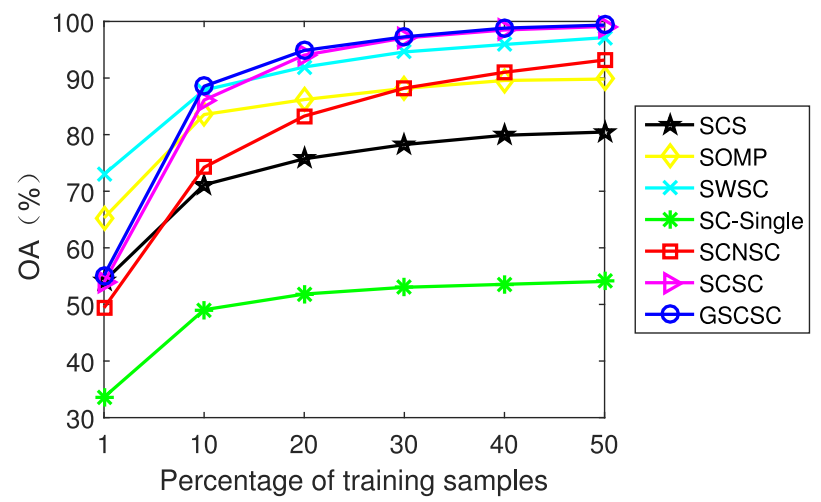

Fig. 10. Classification performance under different training samples.

explore spatial consistency and enforce the spatial structures along the spectral dimension, and thus show many advantages in the classification.

\section{H. Performance Under Different Training Samples}

In this section, we analyze the classification performance of various methods with different sizes of training sets on the Indian pines dataset. We randomly select $1 \%$ to $50 \%$ from each class as the training sets and the rest samples are treated as the testing sets. Our evaluation measure is OA which is calculated from the mean of five-runs. When the number of training samples becomes large, the performance of all methods increases as shown in Fig. 10. The proposed methods have achieved great results and the performance of SCSC and GSCSC increases rapidly to above 95\%. SOMP and SWSC incorporate spatial correlations between pixels and obtain good performance with a very small amount of training samples. SCSC and GSCSC surpass them as the training samples increase. The SC-Single's accuracy is low under different training sizes because only one band of spatial patch is used. It does not capture sufficient spectral distribution of data. SCNSC outperforms SRS and SC-Single by exploring all bands for spatial information. SCSC and GSCSC enforce structural consistency on the model constantly and achieve better performance than SCNSC.

\section{CONCLUSION}

In this paper, we have introduced a novel joint spectralspatial sparse coding model with structural consistency for HSI classification. This method captures the spatially consistent local structures in all bands by enforcing the same coefficient at the same location of different bands during the image reconstruction process. This model also preserves spectral characteristics of different bands by generating different dictionaries. A graph Laplacian sparsity constraints is combined into the proposed method to make the learned sparse coefficients better characterize the relationships between spectral responses. We have validated the effectiveness of the proposed methods on two real-world HSI datasets. The experimental results show that our methods significantly outperform the baseline approach. The proposed method is general in nature and can incorporate other 2-D spatial features for HSI classification. We will explore this direction in the future work.

\section{REFERENCES}

[1] D. A. Landgrebe, Signal Theory Methods in Multispectral remote sensing. Hoboken, NJ, USA: Wiley, 2003.

[2] J. Ham, Y. Chen, M. M. Crawford, and J. Ghosh, "Investigation of the random forest framework for classification of hyperspectral data," IEEE Trans. Geosci. Remote Sens., vol. 43, no. 3, pp. 492-501, Mar. 2005.

[3] F. R. Ratle, G. Camps-Valls, and J. Weston, "Semisupervised neural networks for efficient hyperspectral image classification," IEEE Trans. Geosci. Remote Sens., vol. 48, no. 5, pp. 2271-2282, May 2010.

[4] F. Melgani and L. Bruzzone, "Classification of hyperspectral remote sensing images with support vector machines," IEEE Trans. Geosci. Remote Sens., vol. 42, no. 8, pp. 1778-1790, Aug. 2004.

[5] M. Fauvel, J. Chanussot, and J. A. Benediktsson, "Evaluation of kernels for multiclass classification of hyperspectral remote sensing data," in Proc. IEEE Conf. Acoust., Speech Signal Process., 2006, pp. 813-816.

[6] L. Gao et al.,"Subspace-based support vector machines for hyperspectral image classification," IEEE Geosci. Remote Sens. Lett., vol. 12, no. 2, pp. 349-353, Feb. 2015.

[7] J. Peng, Y. Zhou, and C. L. P. Chen, "Region-kernel-based support vector machines for hyperspectral image classification," IEEE Trans. Geosci. Remote Sens., vol. 53, no. 9, pp. 4810-4824, Sep. 2015.

[8] Y. Chen, N. M. Nasrabadi, and T. D. Tran, "Sparsity-based classification of hyperspectral imagery," in Proc. IEEE Geosci. Remote Sens. Symp., 2010, pp. 2796-2799.

[9] A. S. Charles, B. A. Olshausen, and C. J. Rozell, "Learning sparse codes for hyperspectral imagery," J. Sel. Topics Signal Process., vol. 5, no. 5, pp. 963-978, Sep. 2011.

[10] Y. Chen, N. M. Nasrabadi, and T. D. Tran, "Hyperspectral image classification via kernel sparse representation," IEEE Trans. Geosci. Remote Sens., vol. 51, no. 1, pp. 217-231, Jan. 2013.

[11] J. -H. Yang, L. -G. Wang, and J. -X. Qian, "Hyperspectral image classification based on spatial and spectral features and sparse representation," Appl. Geophys., vol. 11, no. 4, pp. 489-499, Dec. 2014.

[12] X. Sun, Q. Qu, N. M. Nasrabadi, and T. D. Tran, "Structured priors for sparse-representation-based hyperspectral image classification," IEEE Geosci. Remote Sens. Lett., vol. 11, no. 7, pp. 1235-1239, Jul. 2014.

[13] L. He, Y. Li, X. Li, and W. Wu, "Spectral-spatial classification of hyperspectral images via spatial translation-invariant wavelet-based sparse representation," IEEE Trans. Geosci. Remote Sens., vol. 53, no. 5, pp. 26962712, May 2015.

[14] M. E. Midhun, S. R. Nair, V. T. N. Prabhakar, and S. S. Kumar, "Deep model for classification of hyperspectral image using restricted Boltzmann machine," in Proc. Int. Conf. Interdiscip. Adv. Appl. Comput., 2014, pp. 17

[15] Y. Chen, X. Zhao, and X. Jia, "Spectral-spatial classification of hyperspectral data based on deep belief network," IEEE J. Sel. Top. Appl. Earth Observ. Remote Sens., vol. 8, no. 6, pp. 2381-2392, Jun. 2015.

[16] A. Plaza et al.,"Recent advances in techniques for hyperspectral image processing," Remote Sens. Environ., vol. 113, no. 9, pp. S110-S122, Sep. 2009. 
[17] U. Srinivas, Y. Chen, V. Monga, N. M. Nasrabadi, and T. D. Tran, "Exploiting sparsity in hyperspectral image classification via graphical models," IEEE Geosci. Remote Sens. Lett., vol. 10, no. 3, pp. 505-509, May 2013.

[18] Z. tao Qin, W. Yang, R. Yang, X. Zhao, and T. Yang, "Dictionary-based, clustered sparse representation for hyperspectral image classification," $J$. Spectrosc., 2015, Art. no. 678765.

[19] W. Li, Q. Du, F. Zhang, and W. Hu, "Hyperspectral image classification by fusing collaborative and sparse representations," IEEE J. Sel. Topics Appl. Earth Observ. Remote Sens., vol. PP, no. 99, pp. 1-20, 2016.

[20] M. Fauvel, Y. Tarabalka, J. A. Benediktsson, J. Chanussot, and J. C. Tilton, "Advances in spectral-spatial classification of hyperspectral images," Proc. IEEE, vol. 101, no. 3, pp. 652-675, Mar. 2013.

[21] Z. H. Nezhad, A. Karami, R. Heylen, and P. Scheunders, "Fusion of hyperspectral and multispectral images using spectral unmixing and sparse coding," IEEE J. Sel. Topics Appl. Earth Observ. Remote Sens., vol. 9, no. 6, pp. 2377-2389, Jun. 2016.

[22] M. D. Mura, A. Villa, J. A. Benediktsson, J. Chanussot, and L. Bruzzone, "Classification of hyperspectral images by using extended morphological attribute profiles and independent component analysis," IEEE Geosci. Remote Sens. Lett., vol. 8, no. 3, pp. 542-546, May 2011.

[23] P. Ghamisi, M. D. Mura, and J. A. Benediktsson, "A survey on spectralspatial classification techniques based on attribute profiles," IEEE Trans. Geosci. Remote Sens., vol. 53, no. 5, pp. 2335-2353, May 2015.

[24] X. Jia and J. Richards, "Managing the spectral-spatial mix in context classification using Markov random fields," IEEE Geosci. Remote Sens. Lett., vol. 5, no. 2, pp. 311-314, Apr. 2008.

[25] J. Xia, J. Chanussot, P. Du, and X. He, "Spectral-spatial classification for hyperspectral data using rotation forests with local feature extraction and Markov random fields," IEEE Trans. Geosci. Remote Sens., vol. 53, no. 5, pp. 2532-2546, May 2015.

[26] Y. Qian, M. Ye, and J. Zhou, "Hyperspectral image classification based on structured sparse logistic regression and three-dimensional wavelet texture features," IEEE Trans. Geosci. Remote Sens., vol. 51, no. 4, pp. 22762291, Apr. 2013.

[27] J. Liu, Z. Wu, Z. Wei, L. Xiao, and L. Sun, "Spatial-spectral kernel sparse representation for hyperspectral image classification," IEEE J. Sel. Topics Appl. Earth Observ. Remote Sens., vol. 6, no. 6, pp. 2462-2471, Dec. 2013.

[28] Z. He, Q. Wang, Y. Shen, and M. Sun, "Kernel sparse multitask learning for hyperspectral image classification with empirical mode decomposition and morphological wavelet-based features," IEEE Trans. Geosci. Remote Sens., vol. 52, no. 8, pp. 5150-5163, Aug. 2014.

[29] H. Zhang, J. Li, Y. Huang, and L. Zhang, "A nonlocal weighted joint sparse representation classification method for hyperspectral imagery," IEEE $J$. Sel. Topics Appl. Earth Observ. Remote Sens., vol. 7, no. 6, pp. 2056-2065, Jun. 2014.

[30] J. Wang, L. Jiao, H. Liu, S. Yang, and F. Liu, "Hyperspectral image classification by spatial-spectral derivative-aided kernel joint sparse representation," IEEE J. Sel. Topics Appl. Earth Observ. Remote Sens., vol. 8, no. 6, pp. 2485-2500, Jun. 2015.

[31] S. Jia, J. Hu, Y. Xie, L. Shen, X. Jia, and Q. Li, "Gabor cube selection based multitask joint sparse representation for hyperspectral image classification," IEEE Trans. Geosci. Remote Sens., vol. 54, no. 6, pp. 3174-3187, Jun. 2016

[32] Z. Xue, P. Du, J. Li, and H. Su, "Simultaneous sparse graph embedding for hyperspectral image classification," IEEE Trans. Geosci. Remote Sens., vol. 53, no. 11, pp. 6114-6133, Nov. 2015.

[33] J. Wright, A. Y. Yang, A. Ganesh, S. S. Sastry, and Y. Ma, "Robust face recognition via sparse representation," IEEE Trans. Pattern Anal. Mach. Intell., vol. 31, no. 2, pp. 210-227, Feb. 2009.

[34] J. Wright, Y. Ma, J. Mairal, G. Sapiro, T. S. Huang, and S. Yan, "Sparse representation for computer vision and pattern recognition," Proc. IEEE, vol. 98 , no. 6 , pp. 1031-1044, Jun. 2010.

[35] Z. Shu, J. Zhou, P. Huang, X. Yu, Z. Yang, and C. Zhao, "Local and global regularized sparse coding for data representation," Neurocomputing, vol. 175, pp. 188-197, Jan. 2016.

[36] M. Zheng et al., "Graph regularized sparse coding for image representation," IEEE Trans. Image Process., vol. 20, no. 5, pp. 1327-1336, May 2011.

[37] S. Gao, I. W. -H. Tsang, and L. -T. Chia, "Laplacian sparse coding, hypergraph laplacian sparse coding, and applications," IEEE Trans. Pattern Anal. Mach. Intell., vol. 35, no. 1, pp. 92-104, Jan. 2013.

[38] L. Tong, J. Zhou, X. Bai, and Y. Gao, "Dual graph regularized NMF for hyperspectral unmixing," in Proc. Int. Conf. Digit. Image Comput., Tech. Appl., 2014, pp. 1-8.
[39] Z. Shu, J. Zhou, L. Tong, X. Bai, and C. Zhao, "Multilayer manifold and sparsity constrainted nonnegative matrix factorization for hyperspectral unmixing," in Proc. IEEE Conf. Image Process., 2015, pp. 2174-2178.

[40] E. Berg and M. P. Friedlander, "Joint-sparse recovery from multiple measurements," IEEE Trans. Inf. Theory, vol. 56, no. 5, pp. 2516-2527, Apr. 2010.

[41] A. Rakotomamonjy, "Surveying and comparing simultaneous sparse approximation (or group-lasso) algorithms," Signal Process., vol. 91, no. 7, pp. 1505-1526, July 2011 .

[42] G. Liu, Z. Lin, S. Yan, J. Sun, Y. Yu, and Y. Ma, "Robust recovery of subspace structures by low-rank representation," IEEE Trans. Pattern Anal. Mach. Intell., vol. 35, no. 1, pp. 171-184, Jan. 2013.

[43] M. Ye, Y. Qian, and J. Zhou, "Multitask sparse nonnegative matrix factorization for joint spectral-spatial hyperspectral imagery denoising," IEEE Trans. Geosci. Remote Sens., vol. 53, no. 5, pp. 2621-2639, May 2015.

[44] Y. Chen, N. M. Nasrabadi, and T. D. Tran, "Hyperspectral image classification using dictionary-based sparse representation," IEEE Trans. Geosci. Remote Sens., vol. 49, no. 10, pp. 3973-3985, Oct. 2011.

[45] S. Jia, X. Zhang, and Q. Li, "Spectralspatial hyperspectral image classification using $\ell_{1 / 2}$ regularized low-rank representation and sparse representation-based graph cuts," IEEE J. Sel. Top. Appl. Earth Observ. Remote Sens., vol. 8, no. 6, pp. 2473-2484, Jun. 2015.

[46] H. Yuan and Y. Y. Tang, "Sparse representation based on set-to-set distance for hyperspectral image classification," IEEE J. Sel. Topics Appl. Earth Observ. Remote Sens., vol. 8, no. 6, pp. 2464-2472, Jun. 2015.

[47] E. Zhang, L. Jiao, X. Zhang, H. Liu, and S. Wang, "Class-level joint sparse representation for multifeature-based hyperspectral image classification," IEEE J. Sel. Topics Appl. Earth Observ. Remote Sens., vol. PP, no. 99, pp. 1-18, Feb. 2016.

[48] A. S. Farani and H. R. Rabiee, "When pixels team up: Spatially weighted sparse coding for hyperspectral image classification," IEEE Geosci. Remote Sens. Lett., vol. 12, no. 1, pp. 107-111, Jan. 2015.

[49] H. Lee, A. Battle, R. Raina, and A. Y. Ng, "Efficient sparse coding algorithms," in Proc. Adv. Neural Inf. Process. Syst., 2006, pp. 801-808.

[50] R. Fletcher, Practical methods of optimization. Hoboken, NJ, USA: Wiley, 1987.

[51] D. Landgrebe, AVIRIS NW Indiana's Indian Pines 1992 Data Set, 1992. [Online]. Available: https://engineering.purdue.edu/biehl/ MultiSpec/hyperspectral.html.

[52] C. -C. Chang and C. -J. Lin, "LIBSVM: A library for support vector machines," ACM Trans. Intell. Syst. Technol., vol. 2, no. 3, pp. 389-396, 2011.

[53] J. A. Richards, Remote Sensing Digital Image Analysis: An Introduction, 5th ed. New York, NY, USA: Springer, 2013.

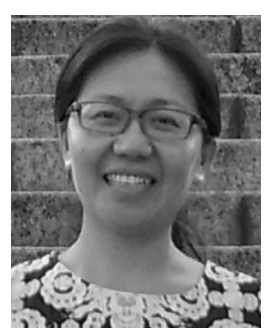

Changhong Liu received the B.S. and M.S. degrees in computer science from Jiangxi Normal University, Nanchang, China, in 2000 and 2004, respectively, and the $\mathrm{Ph} . \mathrm{D}$. degree in computer application technology from University of Science and Technology Beijing, Beijing, China, in 2011.

She is currently a lecturer in the School of Computer and Information Engineering, Jiangxi Normal University, China. During 2015, she was a Visiting Fellow in the School of Information and Communication Technology, Griffith University, Brisbane, QLD, Australia. Her research interests include pattern recognition, hyperspectral imaging, computer vision, and machine learning.

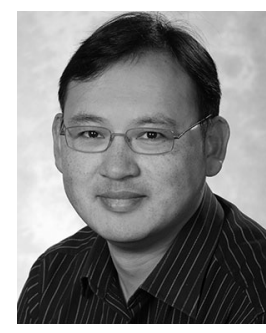

Jun Zhou (SM'12) received the B.S. degree in computer science and the B.E. degree in international business from Nanjing University of Science and Technology, Nanjing, China, in 1996 and 1998, respectively the M.S. degree in computer science from Concordia University, Montreal, QC, Canada, in 2002, and the Ph.D. degree from the University of Alberta, Edmonton, AB, Canada, in 2006.

$\mathrm{He}$ is currently a Senior Lecturer in the School of Information and Communication Technology, Griffith University, Brisbane, QLD, Australia. Previously, he was a Research Fellow in the Research School of Computer Science, the Australian National University, Canberra, ACT, Australia, and a Researcher in the Canberra Research Laboratory, NICTA, Sydney, NSW, Australia. His research interests include pattern recognition, computer vision and spectral imaging with their applications to remote sensing and environmental informatics. 


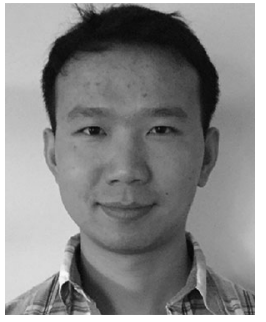

Jie Liang received the B.E. degree in automatic control from National University of Defense Technology, Changsha, China, in 2011. He is currently working toward the $\mathrm{Ph} . \mathrm{D}$. degree in computer vision and pattern recognition at Australian National University, Canberra, Australia.

$\mathrm{He}$ is also a visiting scholar in the School of Information of Communication Technology at Griffith University, Brisbane, QLD, Australia. His research interest includes spectral-spatial feature extraction for hyperspectral image classification.

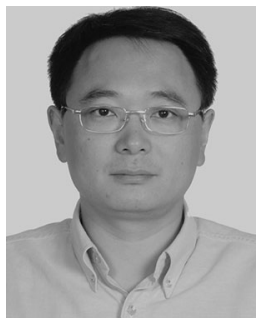

Yuntao Qian (M'04) received the B.E. and M.E. degrees in automatic control from Xi' an Jiaotong University, Xi' an, China, in 1989 and 1992, respectively, and the Ph.D. degree in signal processing from Xidian University, Xi'an, in 1996.

During 1996-1998, he was a Postdoctoral Fellow with the Northwestern Polytechnical University, Xi' an. Since 1998, he has been with the College of Computer Science, Zhejiang University, Hangzhou, China, where he is currently a Professor in computer science. During 1999-2001,2006, 2010, and 2013, he was a Visiting Professor at Concordia University, Hong Kong Baptist University, Carnegie Mellon University, the Canberra Research Laboratory of NICTA, and Griffith University, respectively. His current research interests include machine learning, signal and image processing, pattern recognition, and hyperspectral imaging. Prof. Qian is an Associate Editor of the IEEE Journal of Selected Topics in Applied Earth Observations and Remote Sensing.

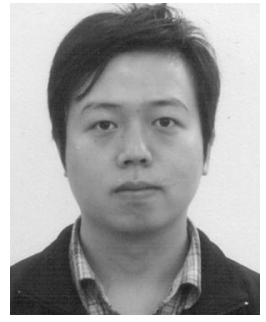

Hanxi Li received the B.Sc. and M.Sc. degrees in electrical engineering from Beihang University, Beijing, China, in 2004 and 2007 respectively, and the $\mathrm{Ph} . \mathrm{D}$. degree in computer science from Australian National University, Canberra, ACT, Australia, in 2011.

He was a Researcher at NICTA, Sydney, NSW, Australia from 2011 to 2015 . He is currently a Special Term Professor in the School of Computer and Information Engineering, Jiangxi Normal University, China. His research interest include visual tracking, face recognition, and deep learning.

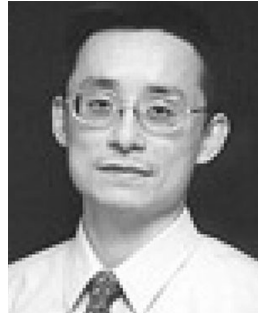

Yongsheng Gao (SM'05) received the B.Sc. and M.Sc. degrees in electronic engineering from Zhejiang University, Hangzhou, China, in 1985 and 1988, respectively, and the Ph.D. degree in computer engineering from Nanyang Technological University, Singapore.

He is currently a Professor with the School of Engineering, Griffith University, Brisbane, QLD, Australia. He had been the Leader of Biosecurity Group, Queensland Research Laboratory, National ICT Australia (ARC Centre of Excellence), a consultant of Panasonic Singapore Laboratories, and an Assistant Professor in the School of Computer Engineering, Nanyang Technological University, Singapore. His research interests include face recognition, biometrics, biosecurity, image retrieval, computer vision, pattern recognition, environmental informatics, and medical imaging. 